\title{
Chinese functional foods and nutraceuticals: plants and products commercialized in the Ciudad Autónoma de Buenos Aires, Argentina
}

\author{
Jeremías P. Puentes ${ }^{1 *}$, Patricia M. Arenas ${ }^{1}$, Julio A. Hurrell ${ }^{1}$
}

\begin{abstract}
Knowledge linked to the traditions of different groups of immigrants in the large cities is a central issue for Urban Ethnobotany, and they constitute a starting point for the discipline approach. This article contributes to the study about local botanical knowledge within the pluricultural context of Buenos Aires-La Plata Metropolitan Area, in particular, the botanical knowledge about plants and its products introduced by Chinese immigrants in the Ciudad Autónoma de Buenos Aires. The registered functional food and nutraceutical plants products marketed by these immigrants (that belong to the Traditional Chinese Phytotherapy) are locally employed for the treatment of some ailments usually linked to the urban lifestyle, such as hypercholesterolemia, anxiety, depression, sexual dysfunction, among others. In this sense, the work contributes to the understanding of the local biocultural diversity (both plants and its associated knowledge). The research followed usual qualitative ethnobotanical methods and techniques, especially semi-structured and free interviews to 250 qualified informants, prior informed consent. In addition, a bibliographic review about species biological activity and studied effects were realized, in order to compare it with the locally assigned uses. An inventory of plant products of 52 vascular plants (vegetables, legumes, fruits, condiments) locally recognized as functional foods was obtained. Plants products belonging to 30 of the 52 treated taxa are commercialized only within the restricted commercial circuit of the Chinese immigrants. Therefore, these taxa are considered "invisible" for the majority of local inhabitants. Plants products of the 22 remaining taxa are marketed in both the restricted Chinese circuit and the general commercial one. Then, these taxa are "visible" for all residents. Local botanical knowledge is evaluated from the circulation of plant products in local trade circuits. "Invisible" taxa may become "visible" when entering the general commercial circuit. This "visualization process" of plants products and its associated knowledge express the local botanical knowledge dynamics.
\end{abstract}

Keywords: Ethnobotany; Urban Pluricultural Context; Local Botanical Knowledge; Chinese Immigration; Argentina.

1 Laboratorio de Etnobotánica y Botánica Aplicada, Facultad de Ciencias Naturales y Museo, Universidad Nacional de La Plata, Calle 64 num. 3, 1900-La Plata, Buenos Aires, Argentina. Consejo Nacional de Investigaciones Científicas y Técnicas, Argentina.

*Corresponding author. $\mathrm{E}$ E-mail address: jeremiasppuentes@gmail.com 


\section{INTRODUCTION}

\section{Urban Ethnobotany}

In the last years, various contributions to Urban Ethnobotany, in different parts of the world, refer to the knowledge linked to the traditions of different immigrant groups and have constituted starting points for the approach of the discipline. In several works, the contribution of the ethnobotanical study in the evaluation of different medicinal plants and products, and its associated knowledge, introduced by immigrants in their new urban context was highlighted. In this framework, ethnomedical studies recorded among immigrants from different urban areas where demonstrated the value of ethnobotanical studies in the analysis of how the Western medical system and other practices of diverse cultures interact in urban pluricultural contexts (Balick et al., 2000; Balick and Lee, 2001; Reiff et al., 2003; Pieroni et al., 2005). Other works evaluate how immigrants adapt to a new cultural context, studying the way of using the medicinal plants linked to the pharmacopeias of their respective countries, recording which ones are still used, which not, and which are the new species incorporated in the urban local scenario (Sandhu and Heinrich, 2005; Ceuterick et al., 2008; Pieroni and Vandebroek, 2009; Volpato et al., 2009; Monteiro et al., 2010; Medeiros et al., 2012; Abreu et al., 2015, among others).

In Buenos Aires-La Plata Metropolitan Area, the Laboratorio de Etnobotánica y Botánica Aplicada (LEBA) has conducted studies on different plants and plant products that are entered into the local urban context by several immigrants segments, analyzing the composition and dynamics of local botanical knowledge (Pochettino et al., 1997, 2008, 2012; Arenas et al., 2011, 2015;
Hurrell and Puentes, 2013, 2017; Hurrell et al., 2013, 2015a, b, 2016, Puentes and Hurrell, 2015; Puentes, 2016, 2017).

\section{Theoretical-methodological framework}

The theoretical-methodological framework of this research based on a broad concept of Urban Ethnobotany understood as the study of the relationships between people and plants in urban pluricultural contexts (Hurrell, 2014; Hurrell and Pochettino, 2014). Urban pluriculturality it is enriched by the increasing presence of diverse immigrants segments which introduce plants and plant products, and its associated knowledge into the local scenario, i.e., respectively "tangible" and "intangible" components (Ladio and Albuquerque, 2016).

The urban botanical knowledge (UBK) constitutes a complex set of knowledge and beliefs about plants, parts thereof, and derivative products. The UBK includes 1) nontraditional knowledge: the taught and learned in educational systems, and the knowledge transmitted by the mass media, specially the Internet (including the scientific knowledge); 2) linked to traditions knowledge: mainly origin traditions of the segments of immigrants, a kind of knowledge that cannot be considered "traditional" because it corresponds to homogeneous cultural contexts (Hurrell, 2014; Hurrell and Pochettino, 2014).

The theoretical-methodological frame also assumes that the UBK is not accessible in a direct way, but can be extrapolated from the "actions" that this knowledge orients, like discourses, practices, strategies of selection, use and consume of plants and its products. At the same time, those actions become evident through the circulation of plant products within the local commercial circuits 
that include the "general" circuit and the "restricted" circuits of diverse immigrants segments. Plant products that circulate inside the general commercial circuit are "visible" for all local urban dwellers (including all the immigrants). Plant products circulating within the restricted commercial circuit of each immigrants segment are visible to members of that particular segment and also some urban residents (non-immigrants and immigrants from others segments) interested in specific plants products. However, the exclusive products of the commercial circuit of a particular immigrants segment are "invisible" for the majority of the inhabitants of local pluricultural context.

At times, some invisible plant products and their associated knowledge become "visible" by entering the general commercial circuit. This becoming is called here "visualization process". This begins when a product of a species restricted to the commercial circuit of the immigrant segments (invisible) is present in the general commercial circuit, especially in health food stores (locally called "dietéticas"). These shops install the product and encourage its consumption, and transmit information about its characteristics, uses and modes of employment (associated knowledge). This first knowledge diffusion is enhanced by the media, the Internet in particular, which plays a fundamental role in knowledge transmission because it acts in a fast way and into multiple directions at the same time. In this context, the media and the "dietéticas" act as true "visualization agents" (Hurrell, 2014; Hurrell and Pochettino, 2014). The distinction between invisible and visible plant species, for the majority of the local urban dwellers, constitutes not only a conceptual distinction but also a methodological tool to address the study of the visualization process, i.e., the local urban botanical knowledge dynamics (Hurrell and Puentes, 2017; Puentes, 2017).

The presence of plant products in local commercial circuits, the general one and the restricted to the immigrants, allows specifying the visibility or invisibility of plant species, and also the visualization of certain species over time. However, although commercial circuits are necessary to assess the invisibility and visibility of plant products, the economic processes and marketing are not sufficient to explain the ethnobotanical context in which "invisibility" or "visibility" has its meaning. In a more complex framework, the commercial circuits are circulation paths of plant products (tangible elements) and, at the same time, these circuits act as communication systems where plant products carry their associated knowledge (intangible elements) that gives them meaning.

\section{Chinese immigration}

This contribution presents the results of ethnobotanical research about functional plant foods introduced and commercialized by Chinese immigrants in the Ciudad Autónoma de Buenos Aires, Argentina. All the species here presented belong to the Traditional Chinese Phytotherapy, in this sense this contribution complements the results obtained in a previous work on that issue (Hurrell and Puentes, 2017). The segment of Chinese immigrants was selected because it is one of the groups of immigrants with the most outstanding presence in the study area, and at present is one of the fast-growing immigrant groups in the country.

From the ethnobotanical point of view, Chinese immigration constitutes an important source of new plant products (food and medicine), especially in the last twenty 
years, which enrich the local botanical knowledge composition.

Argentina received "massive" immigratory waves in the mid-nineteenth century and the first half of the twentieth century. Most of those immigrants were of European origin, especially Italians and Spaniards that settled in a large part of the country. This migration flow has helped to shape the country's cultural heritage, and many current "family traditions" have their roots in that early immigration. In the second half of the twentieth century, a new kind of immigration called "recent" occurred, not massive and localized in the Buenos Aires Metropolitan Area. The Chinese immigration, looking for better economic conditions, is framed into the context of this recent immigration process (Bogado Bordazar 2003; Hurrell and Pochettino, 2014; Hurrell and Puentes, 2017; Puentes, 2017).

\section{Functional foods nutraceuticals}

Plants for "eating and healing" (Etkin and Ross, 1982; Pieroni and Price, 2006; Chen, 2009) are usually considered functional foods and nutraceuticals. "Functional foods" are foods consumed as a source of nutrients and to maintain health or reduce the risk of diseases, with or without knowledge of how or why they have such benefits (Kalra, 2003).

"Nutraceuticals" are functional foods used for the prevention and treatment of diseases, and the consumers know how or why are beneficial to health, e.g., the orange juice. In this context, what it is a functional food for one consumer can act as a nutraceutical for another (Kalra, 2003; Pochettino et al., 2012; Hurrell et al., 2016).

\section{Research objectives}

The basic objective of this contribution is to present for the first time the inventory of species considered functional foods introduced and marketed by Chinese immigrants in the Ciudad Autónoma de Buenos Aires. This inventory represents a descriptive approach that is relevant in that is the necessary condition for an interpretative evaluation of the visibility and invisibility of the Chinese functional foods and their associated knowledge within the local pluricultural context. The study of visualization process implies an original methodological tool with usefulness confirmed in several works carried out in the LEBA in recent years. In this sense, this contribution provides a new background to the urban ethnobotany research and show the importance of immigrant groups in the study of local botanical knowledge.

This contribution includes plant products recognized as functional food and nutraceuticals, some of those taxa are visible to local inhabitants and others are invisible for most of them. In both cases, the new taxa imply a rise in the biocultural diversity within the urban pluricultural context. The research included: 1) an update of the plant products marketed in both commercial circuits, general and restricted to immigrants, and the visibility of the respective species; 2) the registration of the locally assigned uses, both food and medicinal; 3) the information about biological activity and effects studied in the academic field. The first point aims to answer these research questions about the useful species and products: What is the current inventory of the plant species surveyed? Which species are invisible and which are visible? The second and third points aim to answer the questions about the knowledge 
associated with the species: What are the locally assigned uses of the plant species surveyed? Do the assigned uses correspond to the academic research about effects and biological activity of treated species? In short, it is about contributing to the composition and dynamics of local botanical knowledge.

\section{MATERIAL AND METHODS}

\section{Study area and involved actors}

Buenos Aires-La Plata Metropolitan Area has a total area of about 5,000 square kilometers, in which live about 15,000,000 inhabitants (as of 2014). This metropolitan area is the largest in Argentina in both size and population, and the second in South America (after São Paulo Metropolitan Area, Brazil). In this frame, the Ciudad Autónoma de Buenos Aires has 202 square kilometers and about 3,000,000 inhabitants, according to the 2010 National Census (INDEC, 2018). Also according to this census, about 12,000 immigrants were registered for the whole country, about 9,000 from the People's Republic of China, and about 3,000 from Taiwan. Of the total Chinese immigrants in Argentina, 44\% lives in Buenos Aires city, and about $39 \%$ in Buenos Aires province, about $83 \%$ for the Buenos Aires-La Plata Metropolitan Area (Hurrell et al., 2015b; Hurrell and Puentes, 2017).

The most visible presence of Chinese immigrants centered in a sector of Belgrano neighborhood called "Barrio Chino" (Chinatown), where five large supermarkets, various restaurants, and shops were installed, and also cultural events related to Chinese festivities are organized. These characteristics replicate the profile of the Chinatowns in other metropolitan areas of the world (Porterfield, 1951; Sassone and Mera, 2007; Cerrutti, 2009, Hurrell and Pochettino, 2014; Grimson et al. 2016). The five supermarkets offer plant products for the Chinese segment, other immigrants, and also for local residents looking for new products. Towards 2000, the Barrio Chino received about 15,000 visitors every weekend (Bogado Bordazar 2003). Those supermarkets introduce diverse plant products and constitute true dissemination centers for both products and their associated knowledge.

\section{Field works}

\section{1. Ethnobotanical techniques}

The ethnobotanical fieldwork focused on the five large supermarkets in the Barrio Chino (the total of Chinese outlets) to analyze the commercial circuit of immigrants, and 120 health food stores (locally called "dietéticas") of the general commercial circuit to evaluate the visibility of plant species in the local scenario. In total 125 outlets were studied without interruption since 2005. Four visits per year, one for each season, were made to cover all fresh products. The selection of the health food stores started at random and continued until the saturation of information about the investigated plant elements.

The research methodological approach was strictly qualitative, based on usual ethnobotanical techniques like participant observation (interacting with sellers in the plant products survey), free listings, free and semi-structured interviews, applied according to the specific literature (Martin, 1995; Quinlan, 2005; Stepp, 2005; Bernard, 2006; Etkin and Ticktin, 2010; Albuquerque et al., 2014). In particular, semi-structured interviews' questions focused to identify the 
food and medicinal uses of plant products, as well as its diffusion level. With prior informed consent, 250 qualified informants were interviewed (two for each outlet). They are sellers of both sexes and different ages (between 25 and 60 years old), and all of them demonstrated knowledge about the properties of the plant products they sell and guide the consumers on the ways of use.

\subsection{Products and samples}

In all cases, samples were obtained in all the outlets, designated with an alphanumeric code, and deposited in collections of the LEBA. Commercial products, including fragmented plant materials, tinctures, dietary supplements (tablets, capsules), among others, indicate its components in their official labels. When it was necessary, the plant materials were identified based on external morphological characters. For the updated scientific names, The Plant List (2013) and The International Plant Names Index (2015) were consulted. The descriptive and distributional data about the species were obtained from Flora of China (Wu et al., 1995-2013).

Chinese plant products correspond to fruits, vegetables, legumes, and some condiments, whose therapeutic uses belong to the Chinese Traditional Phytotherapy. Except for Coix lacryma-jobi L., valued as functional food, cereals were excluded from this survey due to the great diversity of its products, which will address in the near future.

\subsection{Locally assigned uses}

The "locally assigned uses" (both food and medicinal) were constructed based on data coming from: 1) the interviews; 2) products labels and prospectus; 3) information available in graphic media and Internet, that orients the strategies of selection and consumption of many urban residents interested in obtaining new plant products (Hurrell et al., 2015b; Puentes, 2017). The Information from these sources is mostly coincident and was checked with the available general literature (Zhu, 1998; Yang et al., 2003; Newman, 2004; Hu, 2005; Shi et al., 2005; Hempen and Fischer, 2009; Liu, 2011; Goldberg, 2012; Adams and Lien, 2013; Simoons, 2014; Liu et al., 2015a).

\section{Revision work}

Field works were complemented by a review of the available literature about biological activity and effects evaluated for each treated species. In this regard, we consult various websites, especially PubMed (2018). The search was carried out by scientific name and when necessary keywords such as "biological activity", and "specific effects" (e.g. "sexual dysfunction") according to the assigned local uses. In cases of several references for the same effect, the most current ones were selected. This kind of revision also performed in previous contributions (e.g., Arenas et al., 2015; Hurrell et al., 2015a,b, 2016; Puentes, 2016, 2017; Hurrell and Puentes, 2017). This review becomes pertinent because it is useful information for knowing what uses has academic support, and what uses require validation studies in that context.

\section{RESULTS AND DISCUSSION}

Table 1 summarizes the results obtained for 52 taxa, presented by its scientific name in alphabetical order. For each taxon, the botanical family, geographical distribution, vernacular names, marketed products, and samples were included. The Chinese 
vernacular names appear in bold-italic, the Spanish and English names in italic. Samples codes indicated between brackets, the samples from Barrio Chino in bold.

Locally assigned uses included both food
(F) and medicinal uses (M). The uses in italic correspond to the Chinese Traditional Phytotherapy. Last, the biological activity and effects studied, as well as the respective references, are indicated.

Table 1. Chinese functional foods and nutraceuticals: plants and products commercialized in the Ciudad Autónoma de Buenos Aires, Argentina.

\begin{tabular}{|c|c|}
\hline $\begin{array}{l}\text { SPECIES, FMMLIES, ORGGIN, VERNACULAR } \\
\text { NMMES, PRODUCTS [SAMPIES] }\end{array}$ & $\begin{array}{l}\text { LOCNUY ASSIGNED USES: FOOD AND BIOLOGICAL ACTIVITY AND EVALUATED EFFECTS } \\
\text { MEDICINAL. }\end{array}$ \\
\hline $\begin{array}{l}\text { Allium fistulosum L. } \\
\text { AMAR'LDACEAE } \\
\text { China } \\
\text { Cong bai, negi, cebolla cbina, } \\
\text { cebolla de terdeo, cebolleta, } \\
\text { Japanese buncbing onton, scallion, } \\
\text { Welsb onion } \\
\text { Fresh plants in bundles [RF77] } \\
\text { [RF61] }\end{array}$ & $\begin{array}{l}\text { F. Raw and boiled bulbs and leaves as Antioxidant, anti-aging, anticancer, hypolipidemic, anti- } \\
\text { vegetable and condiment, for salads, hypertensive, antithrombotic, immunostimulant (Lee et } \\
\text { soups, sauces, stewed beef, and al., 2005; Stajner et al., 2006; Aoyama et al., 2008; H. } \\
\text { chicken dishes. } \\
\text { Ueda et al., 2013), anti-inflammatory, analgesic (Wang et } \\
\text { M. Energizing, expectorant, analgesic, al., 2013), antiviral (Lee et al., 2012), antimicrobial (Sohn } \\
\text { antinepbritic, diuretic, cardiovascular et al., 2006), hypoglycemic (Kang et al., 2010), anti- } \\
\text { protective, hypotensive, depurative, obesity (Sung et al., 2018), hepatoprotective (Hwang et } \\
\text { antitumor, anti-insomnia, antidiabetic, al., 2018), cardiovascular protective (Chen et al., 1999). } \\
\text { digestive, antiseptic, vulnerary, } \\
\text { antioxidant. }\end{array}$ \\
\hline $\begin{array}{l}\text { Allium schoenoprasum L. } \\
\text { AMARTLLDMCENE } \\
\text { Eurasia } \\
\text { Xi xiang cong, cebollin, ciboulette, } \\
\text { cbites } \\
\text { Fresh leaves in bundles [B044] } \\
\text { [RF07] } \\
\text { Fresh inflorescences in bundles } \\
\text { [RF08] } \\
\text { Dried leaves in packs [C116] }\end{array}$ & 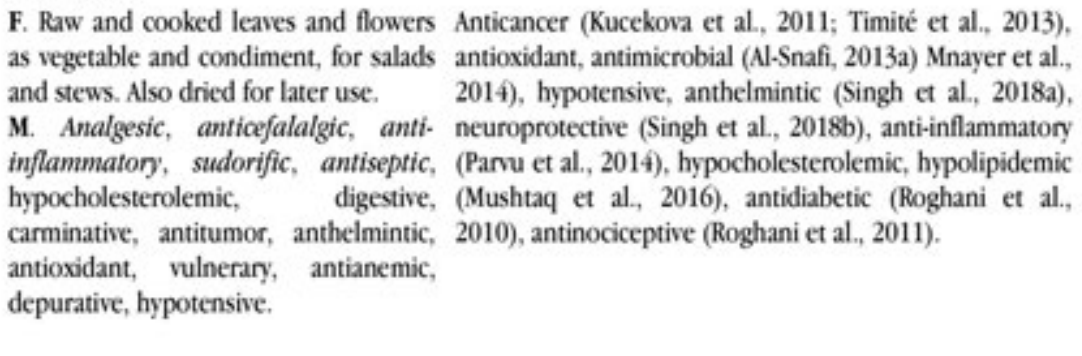 \\
\hline $\begin{array}{l}\text { Allium fuberosum Rottler ex Spreng. } \\
\text { AMAKIuDMCEAF } \\
\text { Warm Asia } \\
\text { Jiu cai, nira, puerno cbino, Cbinese } \\
\text { cbives, Cbinese leek } \\
\text { Fresh leaves in bundles [X034] } \\
\text { Fresh leaves and flower buds in } \\
\text { bundles [F137] }\end{array}$ & 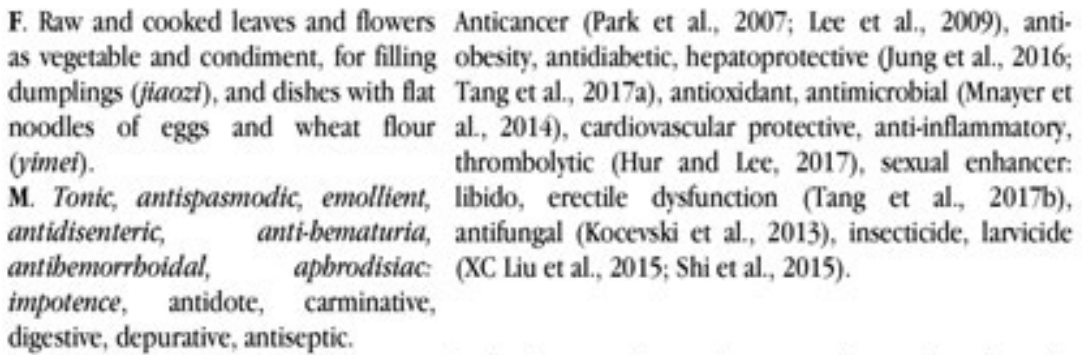 \\
\hline $\begin{array}{l}\text { Apium graveolens L. ['Secalinum' } \\
\text { Group] } \\
\text { AnucEAE } \\
\text { Eurasia } \\
\text { Han qin, apio cbino, Cbinese celery, } \\
\text { leaf celery } \\
\text { Fresh leaves in bundles [F182] }\end{array}$ & 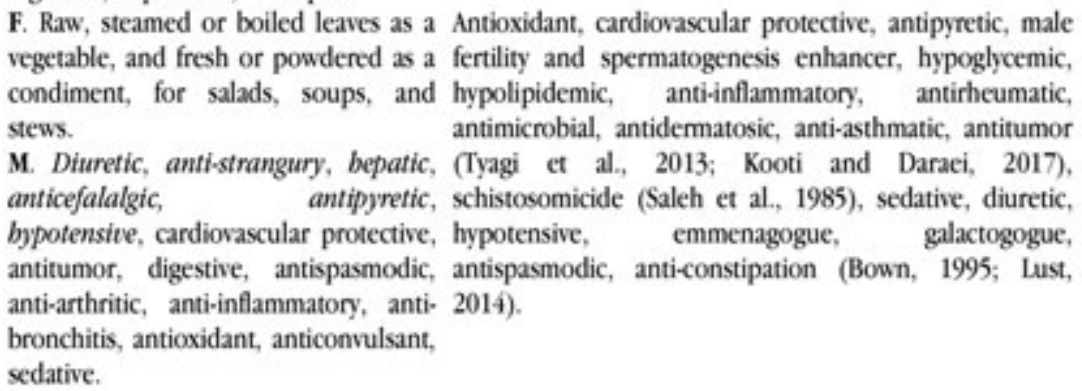 \\
\hline
\end{tabular}


Arctium lappa $\mathrm{L}$.

ASTERMCEN

Eurasia

Niu bang gen, bardana, gobo, burdock

Fresh roots in packs [P325]

Root powder (beverage) [X003]

Dried aerial parts in packs [H282]

Mother tincture [H352]

Armoracia rusticana P.Gaerth., B.Mey. \& Scherb.

BRISSICICEAE

Eurasia

La gen, rábano picante, borseradisb krein

Fresh roots [C131]

Powdered dried roots in packs [C132]

Aterrboa carambola $\mathrm{L}$.

OXUADMCENE

India, China, Philippines, Southeast

Asia

Yang tao, carambola, star fruit Fresh fruits [F156] [F006]

Benincasa bispida (Thunb.) Cogn. CuCuratTACEAE

China, tropical Asia

Dong gua, calabaza cbina, calabaza de invierno, wax gourd, winter melon

Fresh fruits [F183]

Canned juice [BO45]

Solid jam in packs [B046]

\section{Brassica juncea (L.) Czem.}

BRISSICACEAE

Warm Asia

Jie cai, mostaza cbina, broun mustard, Cbinese mustard, leaf mustard

Fresh leaves in bundles [F184]

Pickled leaves in bundles [B048]

Pickled leaves in packs [BO47]

Brassica oleracea L var. albiflora Kuntze

[=B. alboglabra L.H. Bailey]

BRISSICACEAE

China

Gai lan, brócoli cbino, kale china quelan, Cbinese broccoli, Cbinese
F. Boiled, roasted, pickled or stir-fried Anticancer, estrogenic (Feng et al., 2017; Maxwell et al., roots as a vegetable, for dishes with 2017), anti-hyperlipidemic, hepatoprotective, other vegetables and mushrooms, antidiabetic, anti-atherosclerosis, antioxidant (Liu et al., sened with white rice and marinated 2014; Puentes, 2016; Wang et al., 2016; Ahangarpour et chicken, also soups, stews, and tofu al., 2017), anti-inflammatory, anti-arthritic, anti-edema meals. (Maghsoumi-Norouzabad et al., 2016; Carlotto et M. Antitussive, anticefalalgic, anti- al.,2016; Gao et al., 2018), testicular protective (Yari et inflammatory, analgesic, anti-edema, al., 2018), anti-aging (Su and Wink, 2015), anti-allergic antibemorrbotdal, antidermatosic, (Yang et al., 2016), gastroprotective (Li et al., 2016), antinectal prolapse, antirheumatic, anti- hypertensive (Y. Liu et al., 2015b), neuroprotective (Tian arthritic, hepatic, cholagogue, laxative, et al., 2014), antimicrobial (Pereira et al., 2005), digestive, emollient, depurative, schistosomicide, antiviral (Dias et al., 2017).

hypotensive, anti-alopecic, astringent,

nephroprotective, diuretic,

antisyphilitic, sedative, hypoglycemic,

antiseptic, vulnerary.

F. Raw and cooked roots as a Anticancer, antioxidant (Weil et al., 2005; Gafrikova et condiment, for sauces and various al., 2014), anti-inflammatory (Marzocco et al., 2015), dishes, usually as 'wasabi' substitute hypocholesterolemic, gastroprotective (Nguyen et al., [Wasabia japonica (Miq.) Matsum.]. 2013), antimicrobial, spasmolytic (Dekić et al.,2017), M. Dituretic, antirbetumatic, anti- antifungal, insecticide, larvicide (Agneta et al., 2013).

artbritic, cbolagogue, digestive,

urinary antiseptic, analgesic,

antitussive, anti-asthmatic, aphrodisiac:

impotence.

F. Raw or boiled fruits, for cakes, Anticancer, anti-inflammatory, antioxidant, antiulcer, juices, jams, pickles, and chicken antifungal, antimicrobial, antimalarial, meals. hypocholesterolemic, analgesic, hypotensive (Dasgupta M. Antitussive, anti-astbmatic, et al., 2013; Saghir et al., 2013, 2016; Singh et al., 2014; antidermatosic, antipyretic, Leivas et al., 2016; Muthu et al., 2016), hypoglycemic, antimalarial, dituretic, antiseptic, anti-hyperlipidemic (Pham et al., 2017), anti-adipogenic antihemorrhoidal, anti-arthritic, (Rashid et al., 2016).

hypocholesterolemic, anti-

spermatorhoca, cardiovascular protective, hypotensive.

F. Boiled ripe fruits as squash, for Antioxidant, anti-inflammatory, analgesic, hypoglycemic, soups and stuffed with meat, shrimps, muscle relaxant, gastroprotective, anti-asthmatic, and vegetables, also for sauces and diuretic, nephroprotective, anthelmintic, hypoglycemic, confectionery. Raw or pickled young hypolipidemic, antimicrobial, neuroprotective, fruits as a cucumber. antidepressant, anticonvulsant, anxiolytic (Al-Snafi, M. Dituretic, antidiarrbeal, antiulcer, 2013b; X. Jiang et al., 2016), anticancer (Singh et al., expectorant, antibemorrboidal, anti. 2016), hypotensive (Ghelani et al., 2014), antiandrogenic edema, anti-inflammatory, (Nahata and Dixit, 2014), prostatic hyperplasia inhibitor: antidiabetic, tonic, antipyretic, anti- erectile dysfunction (Nandecha et al., 2010), anti-aging asthmatic, hypotensive, cardiotonic, (Sabale et al., 2011), immunostimulant (Une and Doshi, neuprotective: insanity, schizophrenia, 2016).

anticonvulsant, menstrual disorders.

vulnerary, aphrodisiac: impotence.

F. Raw, boiled, stir-fried or pickled Anticancer (Arora et al., 2016; Kwak et al., 2016), leaves as vegetable and condiment, for antiviral (Lee et al., 2014), antifungal (Oguro et al., salads and as a dress for various basic 2014), antibacterial (Engels et al., 2012), antioxidant meals. Crushed seeds to make mustard. (Cartea et al., 2011), anti-obesity, hypotriglyceridemic, Sprouted seeds for salads. hypocholesterolemic (Lee et al., 2018), antiamnesic, M. Analgesic: sore tbroat, lung abscess, antidepressant (Thakur et al., 2014a,b), antidiabetic emollient, anti-bronchitis, anti- (Yadav et al., 2004), antinociceptive (Rahmatullah et al., asthmatic, anti-allergic, diuretic, anti- 2010).

arthritic, antitumor, antirheumatic, antioxidant.

F. Raw, boiled, steamed, stir-fried or Anticancer, antioxidant, anti-allengic, anti-ophthalmic fried shoots with leaves and flowers, Jiao et al., 1998; Liu et al., 2007; Cartea et al., 2011; for various dishes. Anita et al., 2014; Xu, 2018), detoxifying, antiM. Anti-astbmatic, antitussive, inflammatory, antidiabetic, antimicrobial ( $\mathrm{Hu}$ et detoxifying, anti-dipbtberia, al.,2004; Abdulkareem et al., 2017), hypocholesterolemic analgesic, neuroprotective, (La et al., 2013).

antioxidant, hypotensive, antianémico, 
kale

Fresh leaves in bundles [F185]

Brassica rapa L. var. cbinensis (L)

Kitam.

BRISSCACEN

China

Pak choi, bok choi, pai tsai, choy

sum, cbapaicbai, yuchai, col cbina,

Chinese cabbage

['Chinensis' Group]

Fresh plantsin bundles: pak cbot

[RF26] [R005], bolccboi [R030].

paitsai [B061]

['Parachinensis' Group]

Fresh plants in bundles: cboy sum

[B060]

Brassica rapa L. var. glabra Regel

['Pekinensis' Group]

BRISSICACEAE

China

Huang ya bai cai, bakusai, repollo

cbino, Peking cabbage

Fresh plants [RF06] [RF71]

Cinnamomum cassia (L.) J. Pres!

LAITRCEAE

China

Rou gui, canela cbina, Cbinese

cassia, Cbinese cinnamon

Fragmented dried bark in packs

[B001]

Powdered dried bark in packs [H454]

Citrus japonica Thunb.

Rutaceas:

China

Jin gan, quinoto, kumquat

Fresh fruits [B062] [F177]

Fruits preserved in syrup [RF53]

Candied fruits in packs [H117]

Citrus maxima (Burm.) Merr.

RUTACEAE

India, China, Philippines, Southeast

Asia

You, pomelo cbino, pampelmuse, sbaddock

Fresh fruits [RF54]

\section{Citrus medica $\mathrm{L}$.}

RUTACEAE

India, China, Burma

Xiang yuan, cidra, citron

Citron and honey for infusions hypocholesterolemic, cardiovascular protective, antitumor.

F. Boiled, steamed, stir-fried and Anticancer, pulmonary protective, antioxidant, pickled leaves as cabbage, for soups, hepatoprotective, cardiovascular protective (Jiao et al., sauces, stews and meat dishes. Raw 1998; Rochfort et al., 2006; Al-Snafi, 2015), antiyoung leaves for salads. inflammatory, antiplatelet, antimicrobial, M. Anti-inflammatory, anti- immunostimulant, detoxifying, anti-allergic (Cartea et al, constipation, digestive, diuretic, 2011), analgesic, antidepressant (Rahman et al.,2015). antitumor, slimming, antiosteoporosis, antidiarrheal, cardioprotective, hypocholesterolemic, anti-scury, antipyretic, antioxidant, antiseptic, antidiabetic.

F. Boiled, steamed, stir-fried and Antioxidant, anticancer, anti-allergic, cardiovascular pickled leaves as cabbage, for soups, protective, anti-atherosclerosis, antidiabetic, anti-obesity, stews and meat dishes. Raw leaves for anti-inflammatory (Jiao et al., 1998; Cartea et al., 2011; beverages.

Seong et al., 2016; Joo et al., 2017).

M. Diuretic, digestive, anti-

constipation, anti-edema,

antidiarrheal, cardioprotective,

antitumor, anti-inflammatory, antistress.

F. Bark as a spice, for soups, sauces, Anticancer, antioxidant, anti-dyspepsia, antiulcer, stews, and various dishes, also in spice antipyretic, cardiovascular protective, antiplatelet, mixtures (Chinese five-spice powder), hypotensive, anti-obesity, antidiabetic, antiviral, M. Antispasmodic, anti-dyspepsia, antimicrobial, osteoblastic, immunomodulatory, antiantinepbritic, anti-artbritic, analgesic, inflammatory, antidermatosic, anti-dyslipidemic, menstrual disorders, apbrodisiac: hypocholesterolemic, hepatoprotective, impotence, frigidity, antiulcer, nephroprotective, neuroprotective, antidepressant, digestive, carminative, antidiarrheal, anxiolytic, anti-dysmenorrhea, estrogenic, sexual anti-constipation, antipyretic, enhancer: erectile dysfunction (Puentes, 2016; Hurrell hypotensive, anti-edema, and Puentes, 2017; B.Y. Chang et al., 2018; Yun et al., antioxidant,anti-enuresis,anti- $\quad$ 2018).

inflammatory, antidiabetic, antispermatorrhoea, anti-infertility.

F. Raw and cooked fruits preserved in Anticancer, antioxidant, smooth muscle relaxant, uterine syrup, chutney, jam, jelly, candies, contraction stimulant, hypotensive, antimicrobial pickles (acids or sweets), and antiviral (Zhou et al., 2011; Lim 2012b; Dosoky and beverages: liqueurs, infusions. Setzer, 2018), anti-inflammatory, antidermatosic (Yang et M. Expectorant, antitussive, anti- al., 2010), antitussive, expectorant (Gairola et al., 2010), fatigue, resolutive, diuretic, brain cardiovascular protective, anti-obesity, antidiabetic tonic, antitumor, cardiovascular (Aruoma et al., 2012).

protective, antidiabetic, slimming, anti-

inflammatory, antioxidant, anti-aging,

antidermatosic.

F. Raw pulp in salads also preserved in Antioxidant, anti-inflammatory, anti-arthritic, analgesic, jams, jellies, and juices. The peel for muscle relaxant, antidiabetic, anxiolytic, antidepressant, make marmalade, candied or dipped in anticonvulsant, anti-insomnia, antimicrobial, larvicida, chocolate. antihemorrhoidal, hepatoprotective, hypotensive M. Diuretic, anti-constipation, hypocholesterolemic (Vijaylakshmi and Radha, 2015; digestive, detoxifying, brain tonic, Sawant and Panhekar, 2017; Singh and Navneet, 2017), cognitive enhancer: sedative,anti- anticancer (Ademosun et al., 2015), cardiovascular insomnia, anticonvulsant, antioxidant, protective (Buachan et al., 2014), anti-hypenglycemic, anti-asthmatic, antitussive, antiseptic, anti-hyperlipidemic (Nwaka et al., 2014).

antispasmodic, antidiarrheal,

hypocholesterolemic, hypotensive, cardiotonic, anti-arthritic, antidiabetic.

F. Fruits peel (the pulp is usually dry, it Antioxidant, cardioprotective, anti-hypertensive, is not consumed) for jams, sauces, anticancer, antidiabetic, antimicrobial, anthelmintic, dressings, marinades, pickles, and spicy antiviral, diuretic, anticatarrhal, anticefalalgic, antipickles, fish dishes, pastry, constipation, carminative, antiulcer, antispasmodic, anticonfectionery, and beverages: juices arthritic, sedative, antiseptic, analgesic, hypolipidemic, 

expectorant, antispasmodic, neuroprotective, cognitive enhancer (Chhikara et al., analgesic, antiemetic, antidote, 2018).

carminativo, antihemorrhoidal,

Citrus $\times$ microcarpa Bunge $[=\times$ Citrofortunella microcarpa (Bunge) Wijnands; $C$ reticulata Blanco $\times C$ japonica Thunb. ] RUTACEAE

China, Philippines

Jin Ju, calamansi, lima calamansi, calamondin

Fruits in yogurt [X020]

Coix lacryma-jobi $\mathrm{L}$

POACEAE

India, Bhutan, Nepal, Sri Lanka,

China, Southeast Asia, Philippines,

New Guinea

Yi yi ren, lágrimas de Job, Job's tears Dried seeds in packs [H307]

\section{Colocasia esculenta (L) Schot} ARICEAE

India, China, Southeast Asia

Yu tou, taro, dasbeen

Fresh tubers [RF58]

Frozen fragmented tubers [B050]

Tubers and coconut milk (beverage) [B051]

Cucumis melo $\mathrm{L}_{\mathrm{c}}\left[=C_{\text {. }}\right.$ melo var. makuuca Makino] ['Makuwa' Group] Cucurbitaceae

India, East Asia

Tian gua, cbamoe, melón coreano, Korean melon

Fresh fruits [B052]

Fresh sliced fruits in packs [B053]

\section{Curcuma longa L.}

ZINGIBERACEAE

Warm Asia

Jiang buang, curcuma, turmeric Fresh rhizomes [X011]

Fragmented dried rhizomes in packs [C136]

Powdered rhizomes in packs [C036] Capsules [P326]

Cymbopogon citratus (DC.) Stapf anthelmintic, antirheumatic, antiseptic

F. Fruit peel to flavor various dishes, Antioxidant, antimicrobial, anti-aging, hepatoprotective, beverages and infusions. Squeezed anti-hypenglycemic, antidiabetic, nephroprotective,antipulp for making lemonade, cocktails, inflammatory, anti-atherosclerosis, anticancer, ice cream, yogurt, and jams. cardiovascular protective, anticoagulant (Casimiro et al., M. Energizing, antidepressant, 2010; Semaming et al., 2015; Al-Snafi, 2016; M.H. Chen detoxifying (bangover), antacid, et al., 2017; Lou and Ho, 2017).

digestive, emollient, antioxidant,

hypocholesterolemic, antidiabetic, slimming.

F. Boiled seeds as beans, for soups and Antioxidant, immunostimulant, antidiabetic, dishes with rice, cakes, sweets, and hypolipidemic, anti-obesity, anticancer, anti-allergic, antisnacks, also to making liqueurs and inflammatory, anti-fatigue, antinociceptive, antimicrobial, infusions. antiviral, nephroprotective, diuretic, hypouricemic, M. Diuretic, spasmolytic, anti-dysuria, antiprogestogenic, abortive, antiemetic, antisyphilitic, antidiarrbeal, anti-artbritic, gastroprotective, hepatoprotective, anti-dysmenorrhea antirbeumatic, antipyretic, antitumor, (Hurrell and Puentes, 2017; Son et al.,2017). anti-inflammatory, anti-Gatigue,

hypocholesterolemic, anti-obesity.

F. Baked, boiled, steamed, roasted, stir- Anticancer, antimicrobial (Kim et al.,2010; Kundu et al., fried or fried tubers, for soups, stews 2012; Park et al., 2013; Pawar et al., 2018), andvarious dishes, beverages, puddings hypolipidemic (Boban et al., 2006), and a food paste called pot. hypocholesterolemic, antioxidant, hypoglycemic, antiM. Spleen tonic, emollient, inflammatory, cardiotonic (Simsek and Nehir, 2015; Lim, antidiabetic, detaxifying, laxative, 2015; Krishnapriya and Suganthi, 2017), testosterone antidermatosic, vulnerany, anti- and testicular parameters increasing (Ribeiro et al., fatigue, anti-dyspepsia, hepatic, 2018), vulnerany (Gonçalves et al., 2013), antispasmodic, antitumor, astringent, immunomodulatory, hematopoietic (Pereira et al., anti-inflammatory, cardiotonic, anti- 2015).

alopecic, anti-allergic,

immunostimulant.

F. Peeled and sliced fruits for salads, Antidiabetic (Chen and Kang, 2013), anticancer (Kim et desserts, ice creams, and dishes with al., 2009, 2012), antioxidant, anti-inflammatory. rice, meat, and spices. The seeds are analgesic, antiulcer, diuretic, gastroprotective, eaten roasted and contain edible oil. hepatoprotective, anti-obesity, hypolipidemic, M. Diuretic, anti-stranguria, hypocholesterolemic, anti-atherosclerosis, antidisenteric, digestive, sedative, cardioprotective, antiplatelet, anti-hyperglycemic, antidepurative, analgesic, anti-arthritic, hyperthyroidism, mnemonic, neuroprotective, antirheumatic, anti-inflammatory, antimicrobial, anthelmintic (Asif et al., 2014). antipyretic, antidiabetic,

antidermatosic, vulnerary, slimming.

F. Fresh (more aromatic) or dried Anti-inflammatory, antioxidant, anticancer, antidiabetic, (fragmented or powdered) rhizomes, vulnerary, cardiovascular protective, anti-obesity, antias a condiment and food coloring, for atherosclerosis, hypolipidemic, hypocholesterolemic, various meals and pastry. It is an hepatoprotective, hypotensive, nephroprotective, important constituent of the curry neuroprotective, anti-arthritic, anti-ophthalmic, antipowder. Young rhizomes can be eaten osteoporosis, anti-dysmenorrhea, testicular protective, fresh as a spicy vegetable. antidermatosic, anti-aging (Nooralshan and AshkaniM. Antispasmodic, emollient, Esfahani, 2013; Hurrell et al., 2015b; Sundar Dhilip analgesic, anti-artbritic, blood tonic, Kumar et al. 2018, Wojcik et al., 2018), antimicrobial menstrual disorders, anti- (Gupta et al., 2015), cognitive enhancer: mnemonic (Yu inflammatory, antirheumatic, hepatic, et al., 2013), anticonvulsant (Akula and Kulkami, 2014), digestive, carminative, anxiolytic, antidepressant (Ceremuga et al., 2017), sexual hypocholesterolemic, anti-asthmatic, enhancer: erectile dysfunction (Abdel Aziz et al., 2012). antitussive, cardioprotective, antitumor, antidiabetic, antioxidant, antidermatosic, neuroprotective, antidepressant, slimming, aphrodisiac: libido, impotence.

F. Raw or cooked young plants as a Anticancer, antimicrobial, antiviral, antioxidant, 
PONCEAE

Warm Asia and Africa

Xiang mao, citronela, pasto limón, lemon grass

Fresh young plants in bundles [R022] [RFO8]

Fragmented young plants in packs [P240] [H171]

\section{Dimocarpus longan Lour.} SAPINDACENE

India, Sri Lanka, China, Philippines, Southeast Asia, New Guinea

Long yan, long yan rou, longan, ojo de dragón, dragon eye

Fresh fruits [B042]

Canned juice [X004]

Dried fruits in packs [RF74]

Arils presened in syrup [F101]

Arils powder (beverage) [X025]

\section{Dioscorea japonica Thunb.}

DIOSCOREACEAE

China, Korea, Japan

Ri ben sbu yu, yamaimo, ñame, Japanese Yam

Fresh tubers [B043]

\section{Diospyros kaki Thunb. \\ EBENACAE \\ China \\ Sbi, sbi di, caqui, persimmon Fresh fruits [B054] [RF75] \\ Dried fruits in packs [RF76]}

condiment, for salads, soups, sauces, detoxifying, analgesic, anti-rheumatic, antimarinades, stews, pork or chicken inflammatory, cardioprotective, expectorass, ank-flu, dishes, pastry, and confectionery, also antitussive, astringent, diuretic, antiscptic, aratiptate for spices mixtures and infusions. hypotensive, anti-arrhythmia, anai-consagotiost, M. Antirbeumatic, analgesic, gastroprotective, antipyretic, anti-obesis, as:atiabetic, vulnerary, anticefalalgic, antimalarial, hypocholesterolemic, zitistherosctercsis, antidiarrbeal, digestive, antacid, anti-dyslipidemic, anticonvulsant, antrbikorve:s, carminative, analgesic, anti- sedative, anxiolytic, antidepressant, anti-tuberculos $x$, inflammatory, anti-arthritic,depurative, insecticide, repellent (Avoseh et al., 2025 5 ; Ekpenyong at hypotensive, hypocholesterolemic, al., 2015; Hurrell et al., 2015b; Mohanisd et al., 2018). anti-insomnia, anticonvulsant, anxiolytic, sedative, antiseptic, antipyretic, antitumor, detoxifying.

F. Fresh aromatic arils for desserts, Anticancer, immunomodulatory, zatioxidant, sweet-and-sour dishes, soups, stews, antidiabetic (Meng et al., 2014), hypouricersis: ‘sheu et snacks, presenved in syrup, juices, and al., 2016), anti-osteoporosis (S. Presk et a., 2016\%, liqueurs, also dried as raisins, in antimicrobial, antimalarial (Sudjaroen, 33)13; Tseng to Chinese sweet dessert soups. al., 2014), anti-inflammatory (Kunwozaraba ct al., 2016), M. Anti-inflammatory, anti-fatigue antidiarrheal, analgesic, anticonvulsant, sedative, (pbysical and mental), analgesic, anxiolytic (Okuyama et al.,1999; Ripa et al., 2014), anticardiotonic, bloodtonic, anti- fatigue (Zheng et al., 2010), anti-insomnia (Ma et al., insommia, anxiolytic, sedative, 2009), mnemonic (Park et al., 2010).

mnemonic, vasoprotective, antipyretic,

antiseptic, antitumor, anti-aging,

vulnerary, slimming, anthelmintic, immunostimulant.

F. Boiled and steamed tubers as a Antioxidant, anti-inflammatory, anticancer, vegetable, for salads, soups, rice meals, cardioprotective (C.T. Chen et al., 2017; Tsukayama et and various dishes, as a potato al., 2018), immunomodulatory (Lin et al., 2009), substitute. probiotic, gastrointestinal enhancer (Hsu et al., 2006), M. Astringent, antidiarrbeal, anti- hypoglycemic (lvorra et al., 1989), estrogenic (Wu et al., astbmatic,antitussite, antidiabetic, 2005), hypocholesterolemic (Kusano et al., 2016), antinepbritic, anti-inflammatory, anti- neuroprotective, mnemonic, antidepressant (Lee et al., arthritic, hepatic, tonic, sedative, anti- 2013; Jeon et al., 2014).

osteoporosis, antipyretic, antispermatorrhoea, estrogenic.

F. Fresh and dried fruits, for jams, ice Anticancer, anti-inflammatory (Cho et al., 2016; Direito creams, cakes, jellies, juices, and et al., 2017; Park et al., 2017), hypolipidemic, liqueurs. hypocholesterolemic, antidiabetic, hypotensive (Butt et M. Expectorant, antitussive, anti- al., 2015), antioxidant (Matsumura et al., 2016), antiastbmatic, antidiarrbeal, obesity (G.N. Kim et al., 2016), antimicrobial (Morita et antidisenteric, bypotensive, anti- al., 2016), antiviral (K. Ueda et al., 2013), anticoagulant stranguria, antitumor, anti. (Lu et al., 2012), anti-allengic, anti-constipation (Kim et constipation, anti-bematuria, al., 2013), neuroprotective, mnemonic, anti-aging antidiabetic, astringent, antipyretic, (Yokozawa et al., 2014; Forouzanfar et al., 2016). anti-dyspepsia, antihemorrhoidal, sedative, anthelmintic, anti-arrhythmia.

slimming, anti-fertility, contraceptive.

F. Boiled or toasted seeds, for salads, Antioxidant, anti-inflammatory, anticancer, soups, stews, and various dishes. Flour neuroprotective, anti-hypertensive, osteoprotective, for pasta and meat substitutes. Soy menopause symptoms, anti-estrogenic, milk (dou nai) provides proteins and hypocholesterolemic, anti-dyslipidemic, serves to make cheese (tofu). From hepatoprotective, antidiabetic, anti-obesity, antiseeds, it is obtained lecithin, and by asthmatic, anti-infertility, anti-ophthalmic, antimicrobial, fermentation the soy sauce. antiviral (Arenas et al., 2015; Hurrell et al., 2016; M. Tonic, diuretic, antidiarrbeal, Ganesan and $\mathrm{Xu}, 2017$; Juritsch and Moreau, 2018). digestive, antispasmodic, antitumor, hypocholesterolemic, antiseptic, antidysmenorrhea, anti-osteoporosis, antidiabetic, antipyretic, antioxidant, slimming.

F. Dry fruits as spice and mixtures of Antioxidant, anti-inflammatory, analgesic, antimicrobial, spices (Chinese five-spice powder), for sedative, anticancer (Wang et al., 2011; Ritter et al., soups and various dishes, also pastries 2014; Asif et al., 2016; Sun et al., 2016), anti- 
Ba jiao bui xiang, anis estrellado, star anise

Dried fruit in packs [X006] [C056]

Powdered fruits in packs [X007]

[C112]

\section{Ipomoea aquatica Forssk.}

CONVOLLACEAE

India, Pakistan, Bangladesh, Nepal, Sri Lanka, China, Philippines, Southeast

Asia, New Guinea, Australia, Pacific

Islands, Africa, South America

Kong xin cai, weng cai, espinaca

cbina, espinaca de agua, uater spinach

Fresh leaves in bundles [F187]

\section{Kaempferia galangal $\mathrm{L}$.}

ZINGIBERMCEAE

India, China, Southeast Asia

Sban nai, galanga, aromatic ginger.

kencur

Dried rhizomes in packs [B033]

[X012]

Lablab purpureus (L.) Sweet

[= Dolichos lablab $\left.\mathrm{L}_{n}\right]$

LEGUMINOSAE

Africa and Asia

Bian dou, chaucha japonesa, poroto de Egipto, byacintb bean, lablab

bean

Fresh legumes in paks [F188] [Bi04]

Lactuca sativa L. var. angustana

Irish ex Bremer ['Asparagina' Group]

ASTERACEAE

China

Wo ju, lecbuga cbina, lecbuga de tallo, stem lettuce

Fresh plants in bundles [F189]

Pickled stems in packs [P328]

Litcbi cbinensis Sonn.

SAPINDACEAE

China, Philippines, Southeast Asia,

New Guinea

Li zbi, litcbi, lycbee

Arils presenved in syrup [RF98]

Canned juice [R134]

Luffa aegyptiaca Mill.

$\left[=\right.$ L. cylindrica $\left(\mathrm{L}_{-}\right) \mathrm{M}$. Roem.

CUCURETMCEA:

South and Southeast Asia

Si gua, esponja vegetal, pepino and liqueurs (as anise substitute). $\quad$ hyperlipidemic, anti-atherosclerosis (Park et al., 2015), M. Analgesic, antirbeumatic, anti- neuroprotective (Rabelo et al., 2015), galactogogue inflammatory, antispasmodic, (Wang et al., 2015), antidermatosic (Sung et al., 2012a), antinepbritic,diuretic, digestive, central nervous system depressant, anxiolytic (Chouksey antidiarrheal, carminative, anti- et al., 2013), antiviral: HIV (Song et al., 2007). insomnia, sedative, anti-fatigue, expectorant, emmenagogue galactogogue, antioxidant.

F. Raw, boiled, steamed and stir-fried Antioxidant, antidiabetic, anti-obesity, hepatoprotective, leaves, for saladsand dishes with anti-ophthalmic, anticancer, diuretic, antimicrobial, antivegetables, noodles, meat, fish or inflammatory, anti-arthritic, antiulcer, diuretic, antidote, seafood. hypolipidemic, hypocholesterolemic, cognitive M. Diuretic, emollient, anti- enhancer: memory and learning, anxiolytic, bematuria, detoxifying, anticonvulsant (Meira et al., 2012; Manvar and Desai, antibemorrbagic, anti-bemoptisis, 2013; Malakar and Choudhury, 2015), hypotensive anti-icteric, anti-constipation, hepatic, (Khayungamnawee et al., 2018).

anticonvulsant, antidiabetic,

anthelmintic, purgative, hypolipidemic,

hypotensive, vulnerary, antiseptic.

F. Fresh or cooked rhizomes as Antioxidant, anti-inflammatory, analgesic, anticancer, vegetable and as a condiment, for diuretic, anti-hypertensive, anticoagulant, cardiotonic, various dishes. Powdered rhizomes anthelmintic, anti-constipation, antispasmodic, digestive, with rice flour for an herbal beverage. carminative, antipyretic, anti-tuberculosis, antimicrobial, M. Anti-astbmatic,antitussive, antiviral, antiallergic, antidiabetic, vulnerary, antidigestive, spasmolytic, anti- hyperlipidemic, hypocholesterolemic, expectorant, inflammatory, bypotensive, analgesic, antitussive, antidermatosic, anti-obesity, insecticide, antiodontalgic, antidiarrheal, repellent, larvicida, antimalarial, bone protector, carminative, anti-constipation, antirheumatic, anticefalalgic, antiodontalgic, sedative, antihemorrhoidal, antiseptic, vulnerary, anti-insomnia,anxiolytic (Amuamuta et al., 2017; Hurrell antidermatosic, antirheumatic, and Puentes, 2017; Kim et al., 2018).

anticefalalgic, antipyretic, antitumor,

antidepressant, sedative, anti-insomnia,

anti-stress, anxiolytic, slimming.

F. Boiled unripe fruits (legumes) and Antioxidant, anti-inflammatory, antinociceptive, boiled seeds (beans) for various dishes. hypolipidemic, hypocholesterolemic, anticancer, Sprouted seeds in salads. antidiabetic, hepatoprotective, antianemic, antimicrobial, M. Digestive,antidiarrbeal, anti- antimalarial, antiparasitic (Lim, 2012a; Hurrell et al., dyspepsia, diuretic, anti-fatigue, 2016; Al-Snafi, 2017).

antitumor, antispasmodic,caminative,

anthelmintic, antipyretic, antidiabetic,

aphrodisiac.

F. Raw, boiled, roasted, stir-fried or Antioxidant, anti-inflammatory, hypocholesterolemic, pickled stalks and leaves for salads, anticancer, antidiabetic (M.J. Kim et al., 2016), stews, and dishes with fish, chicken, galactogogue, antispasmodic, digestive, diuretic, rice, and eggs. antipyretic, antirheumatic, analgesic, antitussive, M. Diuretic, detoxifying, antidote, sedative, anti-insomia, anxiolytic (Duke and Ayensu, galactogogue, emollient, anti- 1985; Bown, 1995; Lust, 2014).

inflammatory, antispasmodic,

antitumor, analgesic, digestive,

antioxidant, antianemic, sedative, antiinsomnia.

F. Raw and cooked arils for syrup, Anticancer, anti-inflammatory, analgesic, jams, desserts, sauces, pickles, ice immunomodulatory, antimicrobial, antiviral, anti-obesity, cream, and wine. antidiabetic, antipyretic, antioxidant, hepatoprotective M. Analgesic, antispasmodic, liver, (Ibrahim and Mohamed, 2015; Emanuele et al., 2017; stomach, testicles and bernia pain, Man et al., 2017), cardiovascular protective (Y. Chen et antitussive, anti-inflammatory, al., 2017), antithrombotic (Sung et al., 2012b).

antidiabetic, anti-obesity, antidiarrheal,

antioxidant, anti-dysmenorrhea,

antitumor, anti-aging,

F. Raw or cooked unripe fruits as a Antioxidant, anti-inflammatory, anticancer, uterine cucumber, for salads, soups, curries, contraction inducer (childbirth), antimicrobial, and various dishes. Ripe fruits (si gua immunostimulant, bronchodilator, anti-asthmatic, luo) are bitter and very fibrous, not antitussive (Partap et al., 2012; Azeez et al., 2013; edible. Roasted seeds are edible and Sharma et al., 2015; Hlel et al., 2017; Garai et al., 2018), 
esponja, Egyptian cucumber, sponge gourd

Fresh fruits [F190]

\section{Lycium barbarum L.}

SOLANACEAE

China

Gou qi zi, goji, Cbinese uolfberry Dried fruits in packs [R169] [D001] Red tea with goji in packs [X014]

\section{Momordica charantia L \\ CUCURBITMCEAE \\ Pantropical \\ Ku gua, pepino amargo, bitter cucumber, bitter melon, bitter gourd, leprosy gourd \\ Fresh fruits [F191]}

\section{Nelumbo nucifera Gaertn.}

NEUMBONACEA

Siberia, Korea, Japan, China, Nepal, Bhutan, India, Sri Lanka, Pakistan, Philippines, Southeast Asia, Australia. Lian, lian zi, loto sagrado, sacred lotus

Fresh rhizomes in packs [D148] Rhizomes powder (beverage) [X001] Rhizomes and almonds powder (beverage) [X015]

Dried seeds in packs [BH20]

Seeds paste in packs [R028]

Nepbelium lappaceum L.

SAPINDMCEAE

Philippines, Southeast Asia

Hong mao dan, rambután, rambutan

Arils preserved in syrup [F121] contain edible oil.

antiviral: HIV (Ng et al., 2011), anti-ophthalmic (Dubey M. Expectorant, antitussice, anti- et al., 2015), antiulcer, antidiarrheal (Naidu et al., 2014), astbmatic, galactogogue, emollient, antidermatosic, anti-allergic ( $\mathrm{Ha}$ et al., 2015), antipyretic, anti-inflammatory, hypolipidemic, hypocholesterolemic (Thayyil et al., diuretic, hepatic, antihemorrhoidal, 2011), hepatoprotective, vulnerary, hypoglycemic, antitumor, astringent, laxative, analgesic (Sanjaya Kumar and Acharya, 2016). analgesic, uterine contraction, spasmolytic, antirheumatic, cardiotonic, antidermatosic, antiseptic, emmenagogue.

F. Fresh ripe fruits preserved in jams, Adaptogen, cognitive enhancer: memony and learning, creams, yogurt, and juices, also for anxiolytic, antidepressant, neuroprotective, antioxidant, soups, vegetables and meat dishes. hypocholesterolemic, hypolipidemic, cardioprotective, Dried fruits as raisins, for infusions and anti-atherosclerosis (Hurrell et al., 2013, 2015a,b, Hu et alcoholic beverages. al., 2018), anticancer, nephroprotective, hepatoprotective, M. Liver and kidney tonic, depuratite, retinal protector, antidiabetic, anti-obesity, antianti-opbtbalmic, anti-artbritic, male osteoporosis,anti-arthritic, anti-inflammatory, infertility, anti-spermatorrboea, anti. immunomodulatory, anti-aging, male fertility enhancer, insomnia, antidematosic, emollient, sexual enhancer: erectile dysfunction (Puentes, 2016; Gao antianemic, anti-aging analgesic, et al., 2017; Hurrell and Puentes, 2017; Shi et al., 2017; anticefalalgic, antidiabetic, antitussive, J.S. Chang et al., 2018).

vulnerary, antioxidant, adaptogen,

aphrodisiac impotence.

F. Boiled, fried, smoked or stir-fried Antioxidant, anti-inflammatory, antimicrobial, ripe fruits, for soups and dishes with thrombolytic (Hussain et al., 2018), anticancer (Qiu and vegetables, legumes and meats, also Jia, 2014; Ali et al., 2018; Faroogi et al., 2018), pickles and for making infusions. hypolipidemic, hypoglycemic, antidiabetic, anti-obesity M. Antidisenteric, antipyretic, anti. (Yin et al., 2008; Zhou et al., 2016; Jones et al., 2018), opbtbalmic, antiseptic, anti-arthritic (Soo May et al., 2018), antiviral, antidermatosic, detoxifying, anti. immunomodulatory, anthelmintic, hepatoprotective, inflammatory, anti-obesiy, emollient, antilipolytic, antiulcer, anti-fertility (jia et al., 2017), antiviral, antitumor, antidiabetic, neuroprotective (Chen et al., 2018), anti-aging (Cao et antihemorrhoidal, anti-constipation, al., 2018).

anthelmintic, anti-anorexy.

immunomodulatory, anti-fertility,

hypocholesterolemic, anti-aging.

F. Boiled, fried, stir-fried or pickled Anticancer, antioxidant, anti-aging, antipyretic, thizomes as vegetables, for soups, antimicrobial, antiviral: HIV, immunomodulatory, antistews, fish meals, and various dishes. inflammatory, anti-arthritic, anti-atherosclerosis, Raw, boiled, toasted, steamed or antithrombotic, anti-arrhythmia, hypotensive, diuretic, pickled seeds for soups, sauces, antidiarrheal, anti-asthmatic, anti-pulmonary fibrosis, creams, and desserts. neuroprotective, cognitive enhancer: memory and M. Tonic, diuretic,antidiarrbeal, learning, anxiolytic, anticonsulsant, antidepressant, anticancer, anti-inflammatory, sedative, anti-insomnia, gastroprotective, antidiabetic, antidermatosic, hepatoprotective, antidiabetic, hypocholesterolemic, antidote, astringent, antiemetic, hypolipidemic, anti-obesty, anti-fertility, sexual hepatoprotective, anti-dyspepsia, enhancer: erectile dysfunction (Yang et al., 2008; jiang et hipolipidémico, anti-anorexy, al., 2011; Zhou et al., 2013; Paudel and Panth, 2015; antidisenteric, antinephritic, anti- Puentes, 2016; Hurrell and Puentes, 2017; Kumaran et leukorrhea, anti-spermatorrhoea, anti- al., 2018).

hematuria, anti-arrhythmia, anti-

obesity, hypotensive, anti-aging,

antitussive, antipyretic, mnemonic, anti-insomnia, anxiolytic, aphrodisiac: impotence.

F. Fresh arils in salads, also presenved Anticancer (Yuvakkumar et al., 2015), antibacterial in syrup, jellies, jams, compotes. (Yuvakkumar et al., 2014), antiviral (Abdul Ahmad et al. M. Astringent, antidiarrbeal, anti. 2017), anti-obesity (Chung et al., 2018), anti-arthritic dyspepsia, antidisenteric, detoxifying. (Kumar et al., 2012), antioxidant, anti-allergic, sedative, antidiabetic, antidermatosic, anxiolytic, antidepressant (Nethaij et al., 2015; energizing, antipyretic, digestive, anti- Hemández et al., 2017), analgesic, anti-inflammatory, constipation, anthelmintic, antiseptic, antidiabetic, hypocholesterolemic, larvicide, anti-flu, antibacterial, sedative, immunomodulatory, antidiarrheal (Sukmandariet al. hypotensive, cardioprotective, 2017).

slimming. 
Perilla frutescens (L.) Britton LAMUACEA India, Bhutan, Korea, Japan, China, Southeast Asia

Zi su, zi su ye, sbiso, Korean perilla, perilla

Fresh leaves in packs [F149]

Pbyllostadbys bambusoides Siebold \& Zucc. $[=$ P. reticulata $($ Rupr.) $\mathrm{K}$.

Koch]

POACENE

China, Japan

Gui zbu, bambú, bamboo

Fresh sprouts [F132]

Pbyllostachys edulis (Carrière) J Houz. [ = P. pubescens $\mathrm{J}$. Houz. $]$

POACEAE

China

Mao zbu, bambi, moso bamboo

Dried sprouts in packs [X005]

Pickled sprouts [F133] [R045]

Fresh fragmented sprouts (zbu ru)

[X008]

Fresh fragmented sprouts in packs [X030]

Prunus mume (Siebold) Siebold \&

Zucc.

ROSACEAE

Korea, Japan, Southeast Asia

Wu mei, ume, ciruela cbina, ciruela japonesa, ciruela ume, Cbinese plum Pickled fruits in packs [F144]

Dried fruits in packs [F143]

Pyrus pyrifolia (Burm. f.) Nakai
RosicesE
China, Southeast Asia
Li, xue li, pera asiática, pera cbina,
Asian pear, Cbinese pear
Fresh fruits [F157] [X195]
Canned juice [F158]
Dehydrated fragmented fruits in packs
[X024]

Raphanus sativus L. var. longipinnatus L.H. Bailey BRISSICACEAE

East and Southeast Asia

Luo bo, daikon, rábano cbino.

Oriental radisb

Fresh roots [F160] [F214]

Pickled roots in packs [X035]

Saccbarum officinarum $\mathrm{L}$.

POACEAE

Southeast Asia, Pacific Islands

Gan zbe, caña de azicar, sugarcane
F. Fresh leaves for various Chinese and Antioxidant, anti-inflammatory, antimicrobial, antiviral: Japanese dishes (susbi), noodles, HIV, hepatoprotective, anti-allergic, antitussive, meats, and fishmeal, also to make hypotensive, sedative, antidepressant, antipyretic (Zhu, beverages and as a condiment (as basil 1998; Igarashi and Miyazaki, 2013; Bachheti et al., 2014; substitute). Yu et al., 2017), anticancer (He et al., 2015; Abd ElM. Antitussite, anti-astbmatic, anti- Hafeez et al., 2018), neuroprotective, cognitive dyspepsia, antiemetic, anticongestive, enhancer: memory and learning (Lee et al., 2016a,b), anticefalalgic, antipyretic, sudorific, anti-asthmatic (Chen et al, 2015), anti-ophthalmic (J. Kim antidote, tonic, hepatoprotective, anti- et al., 2018), anti-adipogenic (M.J. Park et al., 2016), obesity, antispasmodic, anti-allergic, antidermatosic (Komatsu et al., 2016).

carminative, antiseptic, slimming. sedative.

F. Boiled, roasted and pickled shoots, Antioxidant, anti-inflammatory, anticoagulant, for salads, soups, and various dishes. neuroprotective (Hong et al., 2010), anticonvulsant M. Anti-astbmatic, digestive, bepatic, (Kumar et al., 2011), antibacterial (Kim et al., 2011), anti-bematuria, hypotensive, antidiabetic, anticancer, hypocholesterolemic, analgesic, antidiarrheal, antidisenteric, hypolipidemic, cardiovascular protective (Singhal et al., antitumor, anti-inflammatory, 2013; Pance, 2015).

anticonvulsant, antioxidant, vulnerary,

antiseptic.

F. Boiled, fried, roasted and pickled Antioxidant, anti-inflammatory, antimicrobial, young sprouts, for salads, soups, hypolipidemic, hypocholesterolemic, anticancer, broths, and various dishes. vulnerary (Panee, 2015; Pang and Panee, 2016), antiM. Anticongestive, antitussive, hypertensive (J.S.Kim et al., 2008).

sedative, depurative, digestive, anti-

constipation, anti-insomnia,

hypocholesterolemic, anti-

atherosclerosis, antianemic, anti-

inflammatory, cardiovascular

protective, hypotensive, antitumor,

antioxidant, vulnerary, anxiolytic,

antidepressant, slimming.

F. Fresh fruits preserved in jams and Anticancer (Jeong et al., 2006; Park et al., 2011), juices, also dried and pickled hepatoprotective, anti-inflammatory, antioxidant (Khan (umebosbi) and for make liqueur et al., 2017), immunostimulant (Tsuji et al., 2011), anti(umesbu). $\quad$ allengic (Kono et al., 2018), hypouricemic (Yi et al., M. Antitussite, antidiarrbeal, anti- 2012), gastrointestinal regulator (Lee et al. 2017), bematuria, antidisenteric, antidiabetic (Shin et al., 2013), anti-osteoporosis (Yan et antidiabetic, anti-metrorrbagia, al., 2015), antimicrobial (Mitani et al., 2018), antbelmintic, caminative, neuroprotective (Park et al, 2009; M.S. Kim et al., 2016), antispasmodic, laxative, antacid, anti-fatigue (S. Kim et al., 2008).

cholagogue, antipyretic, vulnerary, anti-

fatigue, detoxifying, anti-aging.

F. Raw or boiled fruits, for salads, Antioxidant, detoxifying, anti-hyperlipidemic, sauces, and sweet-and-sour dishes, also hepatoprotective, antidiabetic, anticancer, diuretic, antipresened in syrup, wine, juices, and asthmatic, antimicrobial, anti-allergic, cardiovascular jams. protective, anti-hypertensive, anti-inflammatory James. M. Antipyretic, detoxifying, anti- Martin et al., 2015; G.H. Jiang et al., 2016; Baniwal and inflammatory, antitussive, Hathan 2017), hypocholesterolemic (Choi et al., 2004), antidiabetic, anti-constipation, neuroprotective (Yoo and Yang, 2012).

hypocholesterolemic, diuretic,

astringent, anti-pharyngitis,

hypotensive, antiseptic, antitumor.

F. Raw or boiled roots as vegetables, Anticancer, diuretic, antinephritic, antihemorrhoidal, for salads, soups, and various dishes. anti-gonorrheal, antisyphilitic, anti-obesity, antiviral, M. Digestive, diuretic, antidiarnbeal, antimicrobial ( $\mathrm{Hu}, 2005$; Koyyati et al., 2016), antispasmodic, antibemorrbagic, antioxidant (Azuma et al., 1999), antidiabetic (Okada and antitussive, antidiabetic, Okada, 2015), vascular protective, anti-hypertensive, antidermatosic, depurative, anti- antithrombotic (Kuroda et al., 2018).

obsesity, antitumor, antiseptic.

F. Sap from stems as a refreshing Antidiabetic, hypocholesterolemic, cardiovascular beverage, and to make syrup, sugar or protective, diuretic, antioxidant, anti-inflammatory, molasses. antithrombotic, hepatoprotective, anticancer, analgesic M. Antidiabetic, detoxifying, anti. (Gobinath et al., 2010; Pallavi et al., 2012; Bucio-Noble 
Fragmented stems [F192]

Scbisandra dbinensis (Turcz.) Baill. SCHISANDRMCEAE

East Asia

Wu wei zi, eschizandra, magnolia berry

Dried fruits in packs [RF59] [P208]

Fruits and honey for infusions [B056] Capsules (mixture) [H323]

\section{Solanum melongena $\mathrm{L}$ \\ SOLANACEAE \\ India, China, Burma \\ Qie zi, berenjena, egeplant \\ Fresh ovoid or rounded fruits (black, white, purple) [B057] [F172] [F173] [F174] \\ Fragmented dried fruits [H21] \\ ['Serpentinum'Group] \\ Fresh long and thin fruits: Cbinese \\ egeplant [F194]}

Syzygium samarangense (Blume)

Merr. \& L.M. Perry

M)RTACEAF:

China, Southeast Asia, New Guinea

Yang pu tao, manzana de Jata, Java apple, jambu samarang, uax jambu Fresh fruits [F177]

Vigna angularis (Willd.) Ohwi \& H. Ohashi

LEgtManosaE

Himalayas, China, Japan

Cbi xiao dou, bong dou, adukt, poroto adzuki, adzuki bean

Dried seeds [X022]

Dried seeds in packs [H450] [BH15]

[H060]

Canned anko [X027]

Vigna radiata (L.) R. Wilczek

LEGIMUNOSIE

India, Sri Lanka, Pakistan, China,

Southeast Asia, Africa

Lu dou, poroto mung, mung bean

Dried seeds [H451] [H059]

Dried seeds in packs [X032]

"Cellophane" noodles [R081]

Sprouted seeds (soybean sprouts) in

packs [X029] [R080]

Vigna unguiculata (L-) Walp subsp. unguiculata

LEGUMINOSAE

Africa and Warm Asia

Jiang dou, caupi, poroto tape, blackeyed bean, goat pea constipation, emollient, antitussive, et al., 2018), antimicrobial (Williams et al., 2016), antianti-fatigue, immunostimulant, antipyretic, 2008; Miraj, 2016). antioxidant, antitumor, antilitic, urinary antiseptic, antihemorrhagic.

F. Fresh or dried ripe fruits for Anticancer, antioxidant, antiviral, antimicrobial, desserts, like raisins, and beverages, immunomodulatory, anti-allergic, anti- inflammatory, also pickled and fermented in wine. cardiovascular protective,cognitive enhancer: memory M. Astringent (pulmonary and and learning, anxiolytic, sedative, anti-insomnia, intestinal), nepbroprotective, anti- antidepressant, neuroprotective, anti-asthmatic, astbmatic, antitussive, anti-dyspnea, antitussive, expectorant, anti-ophthalmic, tonic, anti-fatigue (pbysical and antidermatosic, antidiarrheal, antidiabetic, adaptogen, mental), antidiarrbeal, anti-sudorific, sexual enhancer: erectile dysfunction, anti-spermatorrboea, sedative, anti- gastroprotective,uterotonic, anti-obesity, insomnia, anti-hepatitis, anticefalalgic, hypocholesterolemic, hepatoprotective, detoxifying antiemetic, anxiolytic, aphrodisiac: (Hurrell et al., 2015a,b; Puentes, 2016; Hurrell and premature ejaculation, impotence. $\quad$ Puentes, 2017; Szopa et al., 2017).

F. Boiled, grilled, steamed, fried, Antioxidant, anti-inflammatory, analgesic, roasted or pickledfruits,for soups, hypocholesterolemic, hypolipidemic, neuroprotective, purées, stewed with other vegetables, cardioprotective, hypotensive, antithrombotic, antimeat or fish and various dishes. ophthalmic, immunomodulatory, antimicrobial, antiM. Depurative, carminative, asthmatic, antidiabetic, anti-obesity (Das and Barua, antibemorrboidal, antipyretic, 2013; Gürbüz et al., 2018), anticancer (Friedman, 2015), analgesic, anti-inflammatory, anti- osteoblastic, anti-osteoporosis (Casati et al., 2018), astbmatic, antidisenteric, digestive, antiviral (Di Sotto et al., 2018), anti-amnestic (Manasa hypotensive, anti-obesity,antianemic, and Raju, 2014).

vulnerary, antirheumatic, antidiabetic,

cholagogue, antiseptic, anti-insomnia,

antitumor, diuretic, mnemonic.

F. Fresh fruits presenved in sauces, Anticancer, anti-inflammatory, spasmolytic, antijams, syrups, juice, liqueurs, and wine. hypenglycemic, antidiabetic, hypotriglyceridemic, M. Anti-inflammatory, anti-amnestic, hepatoprotective, analgesic, neuroprotective, cognitive anti-scurvy, anticatarrbal, antiseptic, enhancer: memory and learning (Lim, 2012a; Shen and antispasmodic, antipyretic, Chang, 2013; Shen et al., 2013; Zhang et al., 2016), antidiabetic, digestive, diuretic, antidiarrheal (Ghayur et al., 2006), antioxidant, antitumor, astringent, antidiarrheal, antimicrobial (Simirgiotis et al., 2008; Khandaker et al., anti-constipation, hypotensive, 2015).

hypocholesterolemic, carminative, antitussive, slimming.

F. Boiled seeds like beans for various Antioxidant, anti-inflammatory, hypotensive, dishes, often with rice. A seed paste hypocholesterolemic, hepatoprotective, anticancer, antiboiled with sugar (called anko) is used obesity, antidiabetic, antinephritic, anti-arthritic, antifor desserts, in confectionery and osteoporosis, antibacterial, immunomodulatory (Hurrell pastry products.

M. Diuretic, detoxifying, anti-edema, anti-stranguria, antidisenteric, anti. icteric, anti-inflammatory, antitumor, hypocholesterolemic, antidiabetic, slimming.

F. Boiled seeds for soups, sauces, Antioxidant, cardioprotective, hypotensive, antisnacks, ice cream, bread, biscuits, and inflammatory, anti-arthritic, neuroprotective, anticancer, noodles, also fried with meat or hypocholesterolemic, hepatoprotective, antimicrobial, vegetables. Sprouted seeds are sold as immunomodulatory, antidiabetic, carminative (Hurrell et soybean sprouts.

M. Diuretic, antidiarrbeal, antial., 2016; Hashiguchi et al., 2017; Lopes et al., 2018).

inflammatory, detoxifying, antidiabetic, antispasmodic, digestive, carminative, laxative, antitumor, hypocholesterolemic, antioxidant.

F. Boiled, steamed, fried or fermented Antioxidant, neuroprotective, anti-inflammatory, seeds, for soups, porridges, purées, hypolipidemic, hypocholesterolemic, antistews, and various dishes. Sprouted atherosderosis,anticancer, antidiabetic, antimicrobial, seeds for salads. antiviral, anthelmintic, analgesic, hypotensive, M. Diuretic, antidiarrbeal, anti- cardiovascular protective, thrombolytic, anti-icteric, antileukorrbea, anti-spermatorrboea, constipation, anti-anorexy, anticonvulsant, anxiolytic, 
Dried seeds [H452] [H017]

Dried seeds in packs [X033]

\section{Zingiber officinale Roscoe ZIVGIBERACEAE}

India, China, Southeast Asia Jiang, gan jiang, jengibre, ginger Fresh rhizomes [X013] [R079] Fresh rhizomes in packs [X037] Fragmented dried rhizomes in packs [X010] Powdered rhizomes in packs [X009] [C053]

Fragmented glazed rhizomes [R098] Pickled fragmented rhizomes in packs [X036]

Rhizomes and honey for infusions [B058]

Rhizomes powder (beverage) [X026] Capsules (mixture) [P170] Tablets [R151]

\section{Zizipbus jujube Mill.}

RHAMNACEAE

China

Hong zao, da zao, azufaifo, dátil cbino, jujuba, Cbinese date, jujube Dried fruits in packs [H453] Fruitspowder (beverage) [X002] Fruits and honey for infusions [B059] analgesic, antigonorreic, antidiabetic, menstrual disorders (Hurrell et al.,2016; Aduema et al., antihemorrhoidal,

hypocholesterolemic, antianemic, 2018) 2017; Ibrahim Sayeed et al., 2017; Jayathilake et al. anthelmintic, galactogogue, sedative. anti-insomnia, anxiolytic, antioxidant.

F. Raw, boiled, fried, pickled, stir-fried, Adaptogen, cognitive enhancer: memory and learning dried and powdered rhizomes (more anxiolvtic, antidepressant, anticonvulsant, sedative, aromatic), as spice and mixtures of neuroprotective (Hurrell et al., 2015a; Choi et al., 2018), spices (Chinese five-spice powder), for hypolipidemic, anti-dyslipidemic, hypocholesterolemic salads, curries, chutneys, sauces, (Hurrell et al., 2015b), nephroprotector (Al Hroob et al., snacks, and various dishes, also 2018), antidiabetic (Zhu et al., 2018), anticancer (Zheng pastries, candies, icecreams, syrups, et al., 2016), antitussive (Gairola et al., 2010), antibeverages (ginger ale, ginger beer), inflammatory (Ezzat et al., 2018), cardiovascular liqueurs and cocktails. protective (Rastogi et al., 2017), analgesic (Wilson, M. Expectorant, antitussive, anti- 2015), anti-obesity (Ebrahimzadeh Attari et al., 2018), dyspnea, antiemetic, antipyretic, antirheumatic (Srivastava and Mustafa, 1992), antibemorrbagic, analgesic, antimicrobial, antioxidant (Ghasemzadeh et al., 2018), antispasmodic, anti-inflammatory, hypotensive (Torabi et al., 2017), male and female antiantirheumatic, carminative, digestive, infertility (Hosseini et al., 2016; Yilmaz et al., 2018), anti-flu, antimalarial, antioxidant, sexual enhancer: erectile dysfunction (Alhowiriny et al. antidiabetic, cardiovascular protective, 2013).

hypotensive, antidermatosic,

antitumor, sedative, anti-stress,

slimming, aphrodisiac: impotence.

F. Raw and cooked fruits for jams, Antioxidant, anti-inflammatory, immunostimulant, jellies, sauces, soups, juices, cakes, antinephritic, anticancer, anti-dyslipidemic, puddings, bread, also dried as dates. gastroprotective, anti-constipation, antidiarrheal, M. Tonic, anti-fatigue, sedative, anti- hepatoprotective, antidiabetic, anti-obesity, analgesic, insomnia, anti-anorexy, hypotensive, hematopoietic, anti-allergic, antimicrobial, antidiarrbeal, bypotensive, antiviral, anti-fatigue,cognitive enhancer: memory and antianemic, depurative, anti- learning, sedative, anti-insomnia, anxiolytic, allergic,diuretic, expectorant, anti- anticonvulsant, antidepressant, contraceptive, sexual dyspnea, anti-bronchitis, antidote, enhancer: erectile dysfunction (Mahajan and Chopda, laxative, gastrointestinal protective, 2009; J.Chen et al., 2017, Hurrell and Puentes, 2017; Ji anti-constipation,

hypocholesterolemic, antipyretic,

anxiolytic, mnemonic, slimming, antiaging, aphrodisiac: impotence.

\section{Plants and plant products}

The total of 52 species registered corresponds to 24 botanical families (Figure 1). These species correspond to vegetables, legumes, fruits, and condiments that are locally recognized as functional foods. Of the 52 treated taxa, 29 (55.77\%) corresponds to vegetables and legumes: Allium fistulosum, A. schoenoprasum, A. tuberosum, Apium graveolens 'Secalinum' Group, Arctium lappa, Benincasa hispida, Brassica juncea, $B$. oleracea var. albiflora, $B$. rapa var. chinensis, $B$. rapa var. glabra, Coix lacrymajobi, Colocasia esculenta, Cucumis melo 'Makuwa' Group, Dioscorea japonica,
Glycine max, Ipomoea aquatica, Lablab purpureus, Lactuca sativa var. angustata 'Asparagina' Group, Luffa aegyptiaca, Momordica charantia, Nelumbo nucifera, Perilla frutescens, Phyllostachys bambusoides, P. edulis, Raphanus sativus var. longipinnatus, Solanum melongena, Vigna angularis, $V$. radiata, $V$. unguiculata subsp. unguiculata.

Fruits correspond to 15 taxa that represent $28.85 \%$ of the total: Averrhoa carambola, Citrus japonica, C. maxima, C. medica, $C . \times$ microcarpa, Dimocarpus longan, Diospyros kaki, Litchi chinensis, Lycium barbarum, Nephelium lappaceum, Prunus mume, Pyrus pyrifolia, Schisandra chinensis, Syzygium samarangense, 


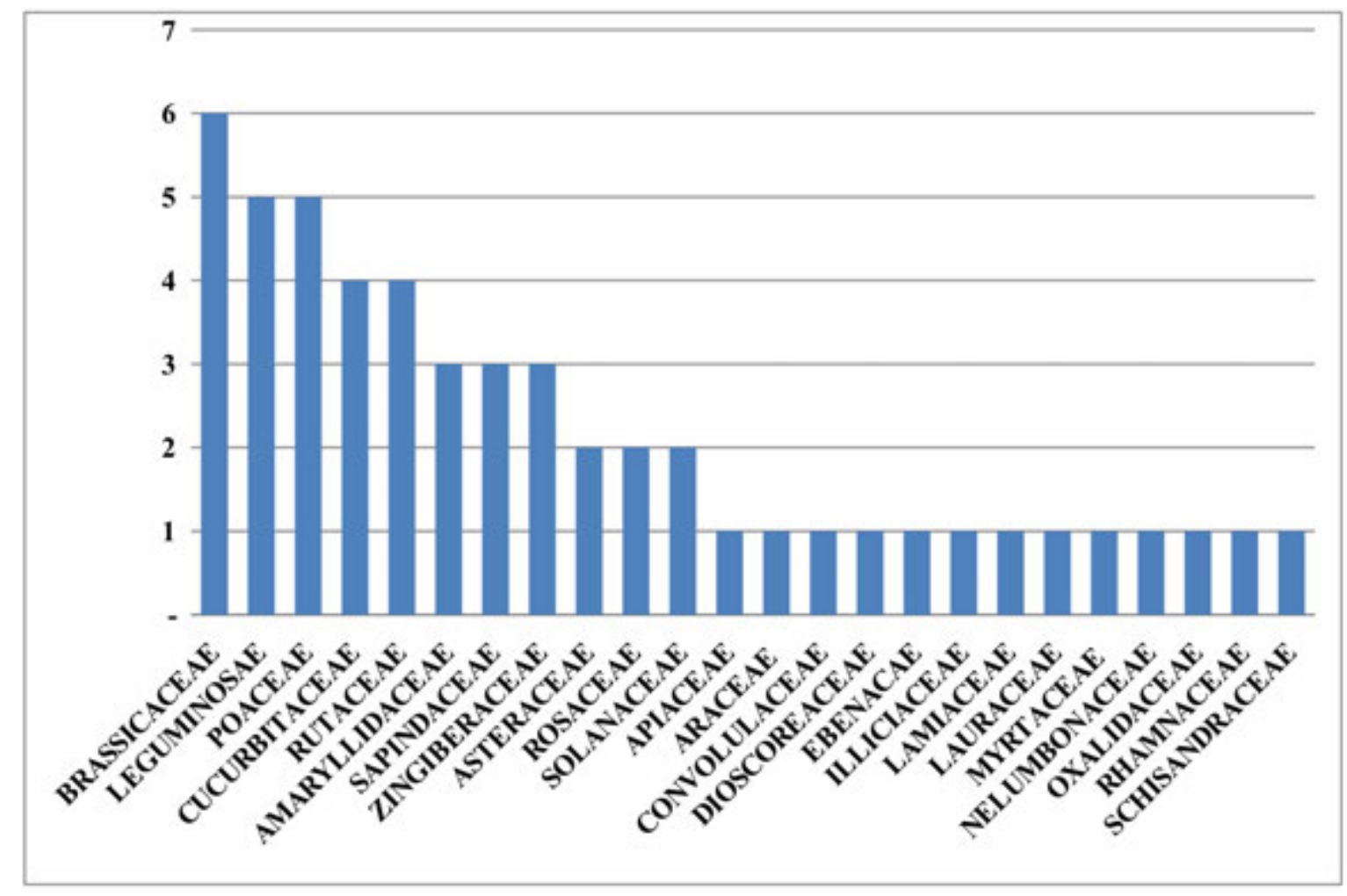

Figure 1. Number of species per botanical families.

Ziziphus jujuba. Last, condiments and flavorings correspond to 8 taxa, $15.38 \%$ of the total: Armoracia rusticana, Cinnamomum cassia, Curcuma longa, Cymbopogon citratus, Illicium verum, Kaempferia galanga, Saccharum officinarum, Zingiber officinale. It is important to note that some species considered as vegetables are as well use as a condiment, e.g., Allium species, Brassica juncea, Perilla frutescens, and some Citrus species.

The plant products commercialized by Chinese immigrants are very diverse. Fresh plants and its parts (like leaves, culms, underground organs, and fruits) proceed from orchards (locally called "quintas"), as affirming the interviewed people of Barrio Chino supermarkets. The orchards are located in periurban areas of the Buenos Aires-La Plata Metropolitan Area, mainly in the sector known as "green belt" or "horticultural belt", near La Plata district. The presence alone of these Chinese crops represents an increase in the metropolitan area agro-biodiversity. In addition, cultivation allows maintaining the knowledge associated with the plants uses (Medeiros et al., 2012). This subject will be a reason for a future contribution. On the other hand, packaged plant products, including fruits and vegetables preserved in syrup, jams, juices and other beverages, also pickled, dried or powdered, are imported from China or other countries. The diversity of products and its associated knowledge represent an increase in local biocultural diversity.

\section{Therapeutic uses}

Table 1 also shows that the locally assigned uses mainly correspond with the biological activity and effects studied. In part, this correspondence is due to the dissemination of scientific knowledge 
through the Internet, which is part of the locally assigned uses construction, what is reflected in the sales arguments of many products (e.g., "The effect of this product is scientifically proven..."). On the other hand, knowledge linked to Chinese tradition is also spread by the Internet as arguments for sale (e.g., "The benefits of this ancestral product...."). Besides, the Traditional
Chinese Phytotherapy as a millenary practice has been an important source of research for the Western science, as show the extensive literature on the subject mentioned before (e.g. Adams and Lien, 2013). With some few exceptions, the academic use categories equal or exceed the locally assigned use categories (Figure 2).

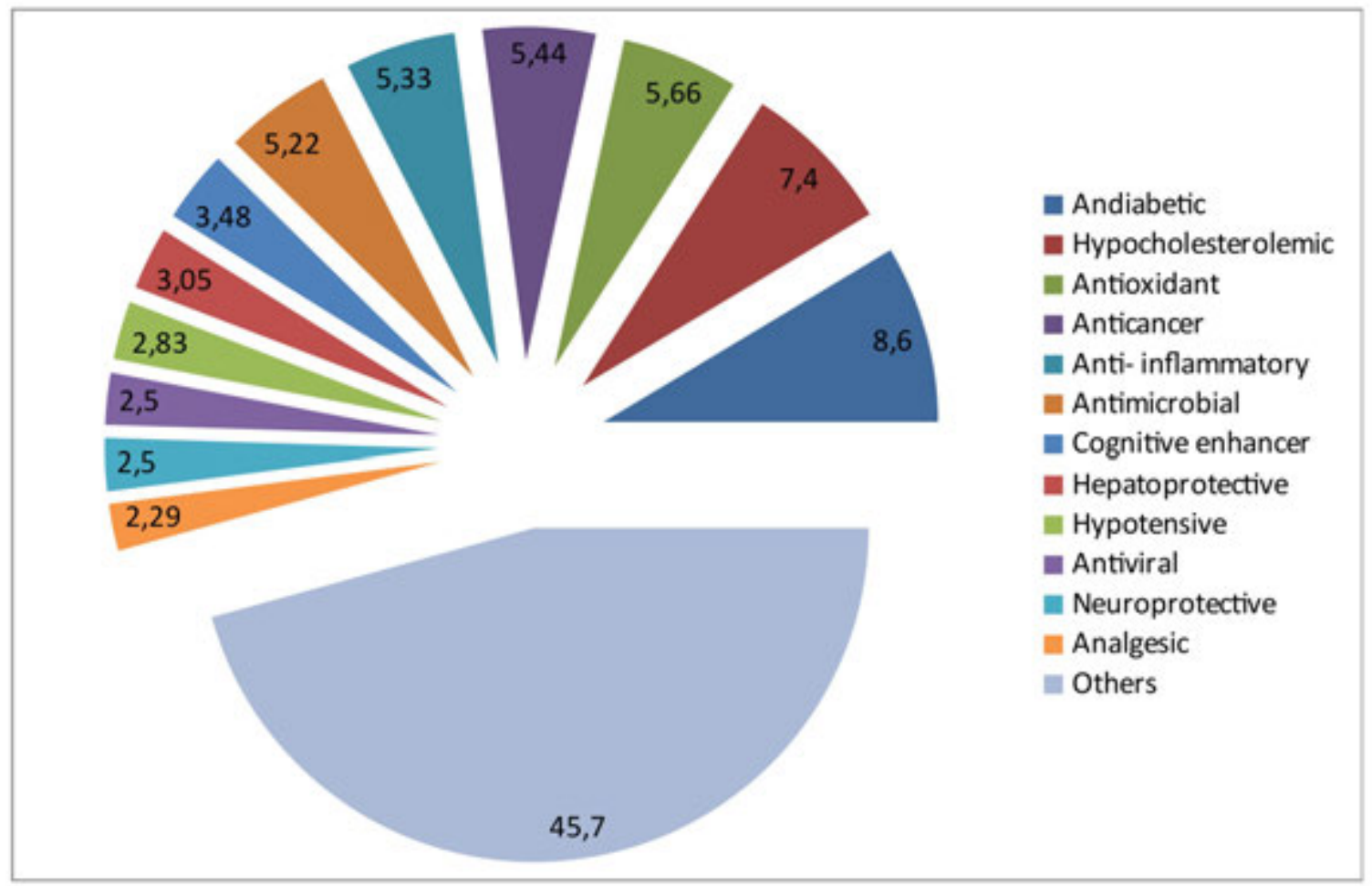

Figure 2. Biological activity and evaluated effects within the academic context.

The relevance of some local uses categories (hypocholesterolemic, anxiolytic, antidepressant, sexual enhancer, among others), reflect the need to respond to health representative problems of the urban lifestyle (Puentes, 2017). In this frame, the academic use categories disseminated by the media (associated with the nontraditional knowledge) guide the selective strategies of plant products by the local urban consumers.

\section{Species visibility}

Of the total of 52 treated species, 30 $(57.69 \%)$ are exclusive of the trade circuit of Barrio Chino, i.e., the species are invisible for most of the local inhabitants: Allium tuberosum, Apium graveolens 'Secalinum' Group, Armoracia rusticana, Benincasa hispida, Brassica juncea, B. oleracea var. albiflora, Cinnamomum cassia, Citrus maxima, C. medica, C. × microcarpa, Coix lacryma-jobi, Colocasia esculenta, Cucumis melo 'Makuwa' Group, Dimocarpus longan, 
Dioscorea japonica, Ipomoea aquatica, Kaempferia galanga, Lactuca sativa var. angustata 'Asparagina' Group, Litchi chinensis, Luffa aegyptiaca, Momordica charantia, Nelumbo nucifera, Nephelium lappaceum, Perilla frutescens, Phyllostachys bambusoides, $P$. edulis, Prunus mume, Saccharum officinarum, Syzygium samarangense, and Ziziphus jujube (Figure 3). Remaining 22 species $(42.31 \%)$ have also products in the general commercial circuit, i.e., the species are visible: Allium fistulosum, A. schoenoprasum, Arctium lappa, Averrhoa carambola, Brassica rapa var. chinensis, B. rapa var. glabra, Citrus japonica, Curcuma longa, Cymbopogon citratus, Diospyros kaki, Glycine max, Illicium verum, Lablab purpureus, Lycium barbarum, Pyrus pyrifolia, Raphanus sativus var. longipinnatus, Schisandra chinensis, Solanum melongena, Vigna angularis, $V$. radiata, V. unguiculata subsp. unguiculata, and Zingiber officinale.

The species "visibility" is a continuum between two extremes: broadly visible (e.g., Glycine max, Lycium barbarum, Zingiber officinale) and scarcely visible (e.g., Brassica rapa var. chinensis, $B$. rapa var. glabra, Lablab purpureus). The case of broad visibility of Lycium barbarum, the "goji", is remarkable. Its presence was registered as a medicinal plant in the local "dietéticas" six years ago (Hurrell et al., 2013). Since then, its diffusion was very fast, mainly enhanced by the Internet.

Visibility is an attribute of the species, although some of its products are invisible. For example, Arctium lappa has exclusive (invisible) products from the Barrio Chino as a functional food and has therapeutic products (herbal materials, mother tinctures) disseminated in the dietéticas of the general commercial circuit (visible). Curcuma longa also has exclusive (invisible) products: the fresh rhizomes from the Barrio Chino, while dried or powdered rhizomes and extract in capsules (dietary supplement) are selling in the dietéticas of the general commercial circuit (visible). In both cases the uses linked to Chinese traditions remain invisible for the majority of local inhabitants; however, the species are visible for the study area context.

The distinction between invisible and visible species products for most of the local inhabitants is a conceptual distinction related to 1) the knowledge "linked to traditions" associated to products of invisible species (circulating in the restricted commercial circuit of Chinese immigrants); and 2) the "nontraditional" knowledge associated to products of visible species (circulating in the general commercial circuit).

The invisible/visible distinction is also a methodological tool, which deals with the study of the visualization process of invisible species that become visible (Hurrell, 2014; Hurrell and Pochettino, 2014; Puentes, 2017). For this contribution, 30 of the 52 taxa treated. This methodological tool also allows evaluating the dynamics of the local botanical knowledge because the visualization implies a contextual change in which the knowledge "linked to traditions" becomes "nontraditional".

\section{CONCLUSIONS}

The Ciudad Autónoma de Buenos Aires constitutes a pluricultural context defined by the coexistence of diverse segments of immigrants, as occurs in the great capitals of the world. In Buenos Aires city is remarkable the recent presence of Chinese immigrants who carry out commercial and cultural activities in a specific city sector called "Barrio Chino". In it, five great supermarkets introduce various plant products linked to 

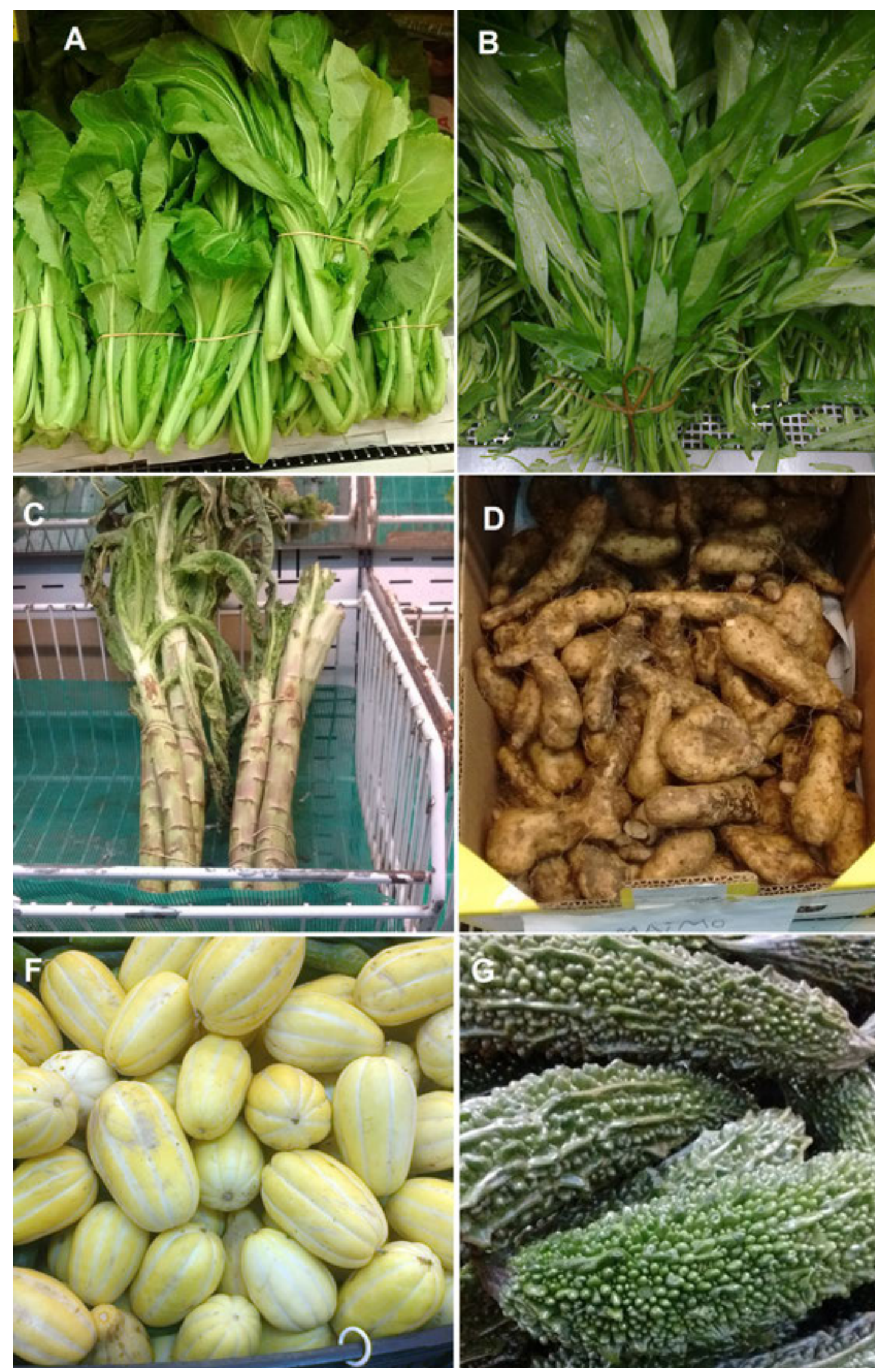

Figure 3. Examples of "invisible" species commercialized in the "Barrio Chino". A. Brassica juncea, Chinese mustard. B. Ipomoea aquatica, water spinach. C. Lactuca sativa var. angustata 'Asparagine', stem lettuce. D. Dioscorea japonica, Japanese yam. E. Cucumis melo 'Makuwa', Korean melon. F. Momordica charantia, bitter cucumber. 
Chinese traditions. In this context, the ethnobotanical research included plants and plant products recognized as functional foods and nutraceutical. The supermarkets of Barrio Chino (restricted commercial circuit) and 120 health food stores (general commercial circuit) were surveyed, with the aim of evaluating the plant products visibility. In total, 52 taxa were found in the Barrio Chino, 30 of which are exclusive to this circuit, and 22 are also marketed in health food stores of the general commercial circuit.

The 52 taxa are represented by plant products that correspond to vegetables, legumes, fruits and condiments, all belonging to the Traditional Chinese Phytotherapy. Food and medicinal locally assigned uses and their biological activity and the studied effects were evaluated. The 30 exclusive taxa of the Barrio Chino are invisible for the majority of local inhabitants. The 22 taxa of both restricted and general trade circuits are visible for all the residents. The methodological tool that implies the distinction between invisible and visible taxa shows that invisible plant products may become visible by entering the general commercial circuit. In this sense, in addition to contributing to the knowledge of new species and their products introduced by Chinese immigrants (that increase the local biocultural diversity), this research contributes to the understanding of the dynamics of local botanical knowledge through the plant products circulation.

\section{ACKNOWLEDGEMENTS}

The authors thank Dr. María Lelia Pochettino and the members of the Laboratorio de Etnobotánica y Botánica Aplicada (LEBA), for their continued collaboration, and all the people who participated disinterestedly in the field works.
This research line is carried out with the financial support of the Universidad Nacional de La Plata and the Consejo Nacional de Investigaciones Científicas y Técnicas (CONICET), Argentina.

\section{REFERENCES}

Abd El-Hafeez AA, Fujimura T, Kamei R (2018) Synergistic tumor suppression by a Perilla frutescens-derived methoxyflavanone and anti-cancer tyrosine kinase inhibitors in A549 human lung adenocarcinoma. Cytotechnology 70:913-919

Abdel Aziz MT, Motawi T, Rezq A (2012) Effects of a water-soluble curcumin protein conjugate vs. pure curcumin in a diabetic model of erectile dysfunction. Journal of Sexual Medicine 9:1815-1833

Abdul Ahmad SA, Palanisamy UD, Tejo BA, Chew MF, Tham HW, Syed Hassan S (2017) Geraniin extracted from the rind of Nephelium lappaceum binds to dengue virus type-2 envelope protein and inhibits early stage of virus replication. Virology Journal 14(1):229, doi: 10.1186/s12985-017-0895-1

Abdulkareem NAO, Periasamy SV, Athinarayanan J, Alshatwi AA (2017) Sulforaphane mitigates cadmium-induced toxicity pattern in human peripheral blood lymphocytes and monocytes. Environmental Toxicology and Pharmacology 55:223-239

Abreu DB, Santoro FR, Albuquerque UP, Ladio AH, Medeiros PM (2015) Medicinal plant knowledge in a context of cultural pluralism: A case study in Northeastern Brazil. Journal of Ethnopharmacology 175:124-130

Adams JD, Lien EJ (2013) Traditional Chinese Medicine: Scientific basis for its use. Royal Society of Chemistry, Cambridge, UK

Ademosun AO, Oboh G, Passamonti S, Tramer F, Ziberna L, Boligon AA, Athayde ML (2015) Inhibition of metalloproteinase and proteasome activities in colon cancer cells by citrus peel extracts. Journal of Basic and Clinical Physiology and Pharmacology 26:471477 
Aduema W, Wariso AC, Agbai JU (2017) Comparative neurobehavioural effect of Vigna unguiculata decrease anxiety related behaviour in Swiss white mice. International Journal of Medical Science and Applied Biosciences 2:97-107

Agneta R, Möllers C, Rivelli AR (2013). Horseradish (Armoracia rusticana), a neglected medical and condiment species with a relevant glucosinolate profile: A review. Genetic Resources and Crop Evolution 60:19231943

Ahangarpour $A$, Heidari $H$, Oroojan AA, Mirzavandi F, Nasr Esfehani K, Dehghan Mohammadi Z (2017) Antidiabetic, hypolipidemic and hepatoprotective effects of Arctium lappa root's hydro-alcoholic extract on nicotinamide-streptozotocin induced type 2 model of diabetes in male mice. Avicenna Journal of Phytomedicine 7:169-179

Akhtar M, Hafeez MA, MuhammadF, ul Haq A, Anwar MI (2008) Immunomodulatory and protective effects of sugarcane juice in chickens against Eimeria infection. Turkish Journal of Veterinary and Animal Sciences $32: 463-467$

Akula KK, Kulkarni SK (2014) Effect of curcumin against pentylenetetrazol-induced seizure threshold in mice: possible involvement of adenosine A1 receptors. Phytotherapy Research 28:714-721

Al Hroob AM, Abukhalil MH, Alghonmeen RD, Mahmoud AM (2018) Ginger alleviates hyperglycemia-induced oxidative stress, inflammation and apoptosis and protects rats against diabetic nephropathy. Biomedicine and Pharmacotherapy 106:381-389

Al-Snafi AE (2013a) Pharmacological effects of Allium species grown in Iraq. An overview. International Journal of Pharmaceuticals and Health Care Research 1:132-147

Al-Snafi AE (2013b) The pharmacological importance of Benincasa hispida. A review. International Journal of Pharmaceutical Sciences and Research 4:165-170.

Al-Snafi AE (2015) The pharmacological importance of Brassica nigra and Brassica rapa grown in Iraq. Journal of Pharmaceutical Biology 5:240-253

Al-Snafi AE (2016) Nutritional value and pharmacological importance of citrus species grown in Iraq. IOSR Journal of Pharmacy 6:76108

Al-Snafi $A E($ 2017) The pharmacology and medical importance of Dolichos lablab (Lablab purpureus): A review. IOSR Journal of Pharmacy 7:22-30

Albuquerque UP, Alves Ramos M, Lucena RFP, Alencar NL (2014) Methods and techniques used to collect ethnobiological data. In: Albuquerque UP, Cruz LVFC, Lucena RFP, Alves RRN (Eds.) Methods and Techniques in Ethnobiology and Ethnoecology. Springer, New York, USA, pp. 14-37

Alhowiriny TA, Al-Rehaily AJ, El Tahir KEH, AlTaweel AM, Perveen S (2013) Molecular mechanisms that underlie the sexual stimulant actions of ginger (Zingiber officinale Roscoe) and garden rocket (Eruca sativa L.). Journal of Medicinal Plant Research 7:2370-2379

Ali MM, Borai HI, Ghanem HM, Abdel-Halim A, Mousa FM (2018) The prophylactic and therapeutic effects of Momordica charantia methanol extract through controlling different hallmarks of the hepatocarcinogenesis. Biomedicine and Pharmacotherapy 98:491-498

Amuamuta A, Plengsuriyakarn T, Na-Bangchang $\mathrm{K}$ (2017) Anticholangiocarcinoma activity and toxicity of the Kaempferia galanga L. rhizome ethanolic extract. BMC Complementary and Alternative Medicine 17(1):213, doi: 10.1186/s12906-017-1713-4

Anita Y, Widiyarti G, Abbas J (2014) Synthesis and elucidation structure of O-para dehydroguaiacol prepared by crude of Brassica oleracea var alboglabra peroxidasecatalyzed oxidation. Journal of Applied Pharmaceutical Science 4:62-65

Aoyama S, Hiraike T, Yamamoto Y (2008) Antioxidant, lipid-lowering and antihypertensive effects of red welsh Onion (Allium fistulosum) in spontaneously hypertensive rats. Food Science and Technology Research 14:99-103

Arenas PM, Cristina I, Puentes JP, Buet Costantino F, Hurrell JA, Pochettino ML (2011) Adaptógenos: plantas medicinales tradicionales comercializadas como suplementos dietéticos en la conurbación Buenos Aires-La Plata (Argentina). Bonplandia (Corrientes) 20:251-264 
Arenas PM, Doumecq MB, Puentes JP, Hurrell JA (2015) Algas y plantas comercializadas como adelgazantes en el Área Metropolitana de Buenos Aires, Argentina. Gaia Scientia 9:32-40

Arora R, Kumar R, Mahajan J, Vig AP, Singh B, Singh B, Arora S (2016) 3-Butenyl isothiocyanate: a hydrolytic product of glucosinolate as a potential cytotoxic agent against human cancer cell lines. Journal of Food Science and Technology 53:3437-3445

Aruoma OI, Landes B, Ramful-Baboolall D, Bourdon E, Neergheen-Bhujun V, Wagner $\mathrm{KH}$ Bahorun T (2012) Functional benefits of citrus fruits in the management of diabetes. Preventive Medicine 54(Suppl.):S12-S16

Asif HM, Akhtar N, Sultana S, Rehman S, Akram M, Rehman G (2014) Medicinal properties of Cucumis melo L. Journal of Pharmacy and Pharmaceutical Sciences 2:58-62

Asif M, Yehya AHS, Al-Mansoub MA, Revadigar V, Ezzat MO, Ahamed MBK, On CE, Murugaiyah V, Majid ASA, Majid AMSA (2016) Anticancer attributes of Illicium verum essential oils against colon cancer. South African Journal of Botany 103:156-161

Avoseh O, Oyedeji O, Rungqu P, Nkeh-Chungag B, Oyedeji A (2015) Cymbopogon species; ethnopharmacology, phytochemistry and the pharmacological importance. Molecules 20:7438-7453

Azeez MA, Bello OS, Adedeji AO (2013) Traditional and medicinal uses of Luffa cylindrica: a review. Journal of Medicinal Plants Studies 1:102-111

Azuma K, Ippoushi K, Ito $\mathrm{H}$, Higashio $\mathrm{H}$, Terao J (1999) Evaluation of antioxidative activity of vegetable extracts in linoleic acid emulsion and phospholipid bilayers. Journal of the Science of Food and Agriculture 79:2010-2016

Bachheti RK, Joshi A, Ahmed T (2014) A phytopharmacological overview on Perilla frutescens. International Journal of Pharmaceutical Sciences Review and Research 26:55-61

Balick MJ, Kronenberg F, Ososki A, Reiff M, Fugh-Berman A, O'Connor B (2000) Medicinal plants used by Latino healers for women's health conditions in New York. Economic Botany 54:344-457
Balick MJ, Lee R (2001) Looking within: urban ethnomedicine and ethnobotany. Alternative Therapies in Health and Medicine 7(4):114-115

Baniwal P, Hathan BS (2017) PhysicoChemical, Nutritional, Functional, Textural and Morphological Characterization of Sand Pear Fruit (Pyrus pyrifolia L.) from Northern Region of India. Asian Journal of Chemistry 29:805-809

Bernard HR (2006) Research methods in anthropology. Qualitative and quantitative approaches. 4th ed. AltaMira Press, Oxford, UK

Boban PT, Nambisan B, Sudhakaran PR (2006) Hypolipidaemic effect of chemically different mucilages in rats: a comparative study. British Journal of Nutrition 96:1021-1029

Bogado Bordazar L (2003) Migraciones internacionales: influencia de la migración china en la Argentina y Uruguay. Tesis Doctoral. Universidad Nacional de La Plata, La Plata, Argentina

Bown D (1995) Encyclopaedia of herbs and their uses. Dorling Kindersley, London, UK

Buachan P, Chularojmontri L, Wattanapitayakul SK (2014) Selected activities of Citrus maxima Merr. fruits on human endothelial cells: enhancing cell migration and delaying cellular aging. Nutrients 6:1618-1634

Bucio-Noble D, Kautto L, Krisp C, Ball MS, Molloy MP (2018) Polyphenol extracts from dried sugarcane inhibit inflammatory mediators in an in vitro colon cancer model. Journal of Proteomics 177:1-10

Butt MS, Sultan MT, Aziz M, Naz A, Ahmed W, Kumar N, Imran M (2015) Persimmon (Diospyros kaki) fruit: hidden phytochemicals and health claims. EXCLI Journal 14:542-561

Cao X, Sun Y, LinY, Pan Y, Farooq U, Xiang L, Qi $J$ (2018) Antiaging of cucurbitane glycosides from fruits of Momordica charantia $L$. Oxidative Medicine and Cellular Longevity 2018:1538632, doi: 10.1155/2018/1538632

Carlotto J, de Souza LM, Baggio CH,Werner MF, Maria-Ferreira D, Sassaki GL, lacomini M, Cipriani TR (2016) Polysaccharides from Arctium lappa L.: Chemical structure and biological activity. International Journal of Biological Macromolecules 91:954-960

Cartea ME, Francisco M, Soengas P, Velasco P (2011) Phenolic compounds in Brassica vegetables. Molecules 16:251-280 
Casati L, Pagani F, Fibiani M, Lo Scalzo R, Sibilia $\mathrm{V}$ (2018) Potential of delphinidin-3-rutinoside extracted from Solanum melongena $L$. as promoter of osteoblastic MC3T3-E1 function and antagonist of oxidative damage. European Journal of Nutrition 2018, doi: 10.1007/s00394018-1618-0

Casimiro MF, Gutierrez M, Leano DR (2010) Evaluation of the hepatoprotective activity of Citrus microcarpa Bunge (Family Rutaceae) fruit peel against acetaminophen-induced liver damage in male BFAD-Sprague Dawley rats. International Journal of Chemical and Environmental Engineering 1:127-132

Ceremuga TE, Helmrick K, Kufahl Z, Kelley J, Keller B, Philippe F, Golder J, Padrón G (2017) Investigation of the anxiolytic and antidepressant effects of curcumin, a compound from Turmeric (Curcuma longa), in the adult male Sprague-Dawley rat. Holistic Nursing Practice 31:193-203

Cerrutti M (2009) Diagnóstico de las poblaciones de inmigrantes en la Argentina. Dirección Nacional de Población, Ministerio del Interior, Buenos Aires, Argentina

Ceuterick M, Vandebroek I, Torrya B, Pieroni A (2008) Cross-cultural adaptation in urban ethnobotany. The Colombian folk pharmacopoeia in London. Journal of Ethnopharmacology 120(3):342-359

Chang BY, Kim DS, Kim HS, Kim SY (2018) Evaluation of estrogenic potential by herbal formula, HPC 03 for in vitro and in vivo. Reproduction 155:105-115

Chang JS, Lee YJ, Wilkie DA, Lin CT (2018) The neuroprotective and antioxidative effects of submicron and blended Lycium barbarum in experimental retinal degeneration in rats. Journal of Veterinary Medical Science 80:11081115

Chen CT, Wang ZH, Hsu CC, Lin HH, Chen JH (2017) Taiwanese and Japanese yam (Dioscorea spp.) extracts attenuate doxorubicin-induced cardiotoxicity in mice. Journal of Food and Drug Analysis 25:872-880

Chen HS, Chen X, Li WT, Shen JG (2018) Targeting RNS/caveolin-1/MMP signaling cascades to protect against cerebral ischemia-reperfusion injuries: potential application for drug discovery. Acta Pharmacologica Sinica 39:669-682
Chen J, Liu X, Li Z, Qi A, Yao P, Zhou X, Dong TX, Tsim KW (2017) A review of dietary Ziziphus jujuba fruit (Jujube): Developing health food supplements for brain protection. Evidence-Based Complementary and Alternative Medicine https://doi.org/10.1155/2017/3019568 2017:3019568,

Chen JH, Tsai SJ, Chen HI (1999) Welsh onion (Allium fistulosum L.) extracts alter vascular responses in rat aortae. Journal of Cardiovascular Pharmacology 33:515-520

Chen L, Kang YH (2013) In vitro inhibitory effect of oriental melon (Cucumis melo L. var. makuwa Makino) seed on key enzyme linked to type 2 Diabetes: Assessment of antidiabetic potential of functional food. Journal of Functional Foods 5:981-986

Chen MH, Yang KM, Huang TC, Wu ML (2017) Traditional small-size Citrus from Taiwan: Essential oils, bioactive compounds and antioxidant capacity. Medicines (Basel) 4:28, doi: 10.3390/medicines4020028

Chen ML, Wu CH, Hung LS, Lin BF (2015) Ethanol extract of Perilla frutescens suppresses allergen-specific Th2 responses and alleviates airway inflammation and hyperreactivity in ovalbumin-sensitized murine model of asthma. Evidence-Based Complementary and Alternative Medicine 2015:324265, doi: 10.1155/2015/324265

Chen NN (2009) Food, Medicine, and the Quest for Good Health: Nutrition, Medicine and Culture. Columbia University Press, New York, USA

Chen Y, Li H, Zhang S, Yang C, Mai Z, Hu X, Gao Z, Deng H (2017) Anti-myocardial ischemia effect and components of Litchi pericarp extracts. Phytotherapy Research 31:1384-1391

Chhikara N, Kour R, Jaglan S, Gupta P, Gat Y, Panghal A (2018) Citrus medica: nutritional, phytochemical composition and health benefits. A review. Food and Function 9:19781992

Cho YH, Kim NH, Khan I, Yu JM, Jung HG, Kim $\mathrm{HH}$, Jang JY, Kim HJ, Kim DI, Kwak JH, Kang SC, An BJ (2016) Anti-inflammatory potential of quercetin-3-O- $\beta$-D-("2"-galloyl)glucopyranoside and quercetin isolated from Diospyros kaki calyx via suppression of MAP signaling molecules in LPS-induced RAW 264.7 Macrophages. Journal of Food Science 81:C2447-C2456 
Choi HJ, Park JH, Han HS, Son JH, Choi C (2004) Effect of polyphenol compound from Korean Pear (Pyrus pyrifolia) on lipid metabolism. Journal of the Korean Society of Food Science and Nutrition 33:299-304

Choi JG, Kim SY, Jeong M, Oh MS (2018) Pharmacotherapeutic potential of ginger and its compounds in age-related neurological disorders. Pharmacology and Therapeutics 182:56-69

Chouksey D, Upmanyu N, Pawar RS (2013) Central nervous system activity of IIlicium verum fruit extracts. Asian Pacific Journal of Tropical Medicine 6:869-875

Chung APYS, Gurtu S, Chakravarthi S, Moorthy M, Palanisamy UD (2018) Geraniin protects high-fat diet-induced oxidative stress in Sprague Dawley Rats. Frontiers in Nutrition 5:17, doi: 10.3389/fnut.2018.00017

Das M, Barua N (2013) Pharmacological activities of Solanum melongena Linn. (Brinjal plant). International Journal of Green Pharmacy 7:274-277

Dasgupta P, Chakraborty P, Bala NN (2013) Averrhoa carambola: An updated review. International Journal of Pharma Research and Review 2:54-63

Dekić MS, Radulović NS, Stojanović NM, Randjelović PJ, Stojanović-Radić ZZ, Najman S, Stojanović S (2017) Spasmolytic, antimicrobial and cytotoxic activities of 5-phenylpentyl isothiocyanate, a new glucosinolate autolysis product from horseradish (Armoracia rusticana P. Gaertn., B. Mey. \& Scherb., Brassicaceae). Food Chemistry 232:329-339

Di Sotto A, Di Giacomo S, Amatore D, Locatelli M, Vitalone A, Toniolo C, Rotino GL, Lo Scalzo R, Palamara AT, Marcocci ME, Nencioni L (2018) A polyphenol rich extract from Solanum melongena L. DR2 peel exhibits antioxidant properties and anti-Herpes Simplex Virus Type 1 activity in vitro. Molecules 23(8):E2066, doi: 10.3390/molecules23082066

Dias MM, Zuza O, Riani LR, de Faria Pinto P, Pinto PLS, Silva MP, de Moraes J, Ataíde ACZ, de Oliveira Silva F, Cecílio $A B$, Da Silva Filho $A A$ (2017) In vitro schistosomicidal and antiviral activities of Arctium lappa L. (Asteraceae) against Schistosoma mansoni and Herpes simplex virus-1. Biomedicine and Pharmacotherapy 94:489-498
Direito R, Lima A, Rocha J, Ferreira RB, Mota J, Rebelo P, Fernandes A, Pinto R, Alves P, Bronze R., Sepodes B, Figueira ME (2017) Dyospiros kaki phenolics inhibit colitis and colon cancer cell proliferation, but not gelatinase activities. Journal of Nutritional Biochemistry 46:100-108

Dosoky NS, Setzer WN (2018) Biological activities and safety of Citrus spp. essential oils. International Journal of Molecular Sciences 19(7):1966, doi: 10.3390/ijms19071966

Dubey S, Saha S, Kaithwas G, Saraf SA (2015) Effect of standardized fruit extract of Luffa cylindrica on oxidative stress markers in hydrogen peroxide induced cataract. Indian Journal of Pharmacology 47:644-648

Duke JA, Ayensu ES (1985) Medicinal Plants of China. Reference, Algonac, USA

Ebrahimzadeh Attari V, Malek Mahdavi A, Javadivala Z, Mahluji S, Zununi Vahed S, Ostadrahimi A (2018) A systematic review of the anti-obesity and weight lowering effect of ginger (Zingiber officinale Roscoe) and its mechanisms of action. Phytotherapy Research 32:577-585

Ekpenyong CE, Akpan E, Nyoh A (2015) Ethnopharmacology, phytochemistry, and biological activities of Cymbopogon citratus (DC.) Stapf extracts. Chinese Journal of Natural Medicines 13:321-337

Emanuele S, Lauricella M, Calvaruso G, D'Anneo A, Giuliano M (2017) Litchi chinensis as a functional food and a source of antitumor compounds: An overview and a description of biochemical pathways. Nutrients 9(9):992, doi: 10.3390/nu9090992

Engels C, Schieber A, Gänzle MG (2012) Sinapic acid derivatives in defatted Oriental mustard (Brassica juncea L.) seed meal extracts using UHPLC-DAD-ESI-MSn and identification of compounds with antibacterial activity. European Food Research and Technology 234:535-542

Etkin NL, Ross PJ (1982) Food as medicine and medicine as food: an adaptive framework for the interpretation of plant utilization among the Hausa of northern Nigeria. Social Science and Medicine 16:1559-1573

Etkin NL, Ticktin T (2010) Advancing an ethnoecological perspective that integrates theory and method in ethnobotany. In: Albuquerque, U.P., Hanazaki, N. (Eds) Recent Developments and Case Studies in Ethnobotany. SBEE/NUPEEA, Recife, Brazil, pp. 33-57 
Ezzat SM, Ezzat MI, Okba MM, Menze ET, Abdel-Naim AB (2018) The hidden mechanism beyond ginger (Zingiber officinale) potent in vivo and in vitro anti-inflammatory activity. Journal of Ethnopharmacology 214:113-123

Farooqi AA, Khalid S, Tahir F, Sabitaliyevich UY, Yaylim I, Attar R, Xu B (2018) Bitter gourd (Momordica charantia) as a rich source of bioactive components to combat cancer naturally: Are we on the right track to fully unlock its potential as inhibitor of deregulated signaling pathways. Food and Chemical Toxicology 119:98-105

Feng T, Cao W, Shen W, Zhang L, Gu X, Guo Y, Tsai HI, Liu X, Li J, Zhang J, Li S, Wu F, Liu Y (2017) Arctigenin inhibits STAT3 and exhibits anticancer potential in human triple-negative breast cancer therapy. Oncotarget 8:329-344

Forouzanfar F, Torabi S, Askari VR, Asadpour E, Sadeghnia HR (2016) Protective effect of Diospyros kaki against Glucose-OxygenSerum Deprivation-Induced PC12 Cells injury. Advances in Pharmacological Sciences 2016:3073078, doi: 10.1155/2016/3073078

Friedman M (2015) Chemistry and anticarcinogenic mechanisms of glycoalkaloids produced by eggplants, potatoes, and tomatoes. Journal of Agricultural and Food Chemistry 63:3323-3337

Gafrikova M, Galova E, Sevcovicova A, Imreova P, Mucaji P, Miadokova E (2014) Extract from Armoracia rusticana and its flavonoid components protect human lymphocytes against oxidative damage induced by hydrogen peroxide. Molecules 19:3160-3172

Gairola S, Gupta V, Bansal P, Singh R, Maithani M (2010) Herbal antitussive and expectorants. A review. International Journal of Pharmaceutical Sciences Review and Research 5(2):5-9

Ganesan K, Xu B (2017) A critical review on polyphenols and health benefits of black soybeans. Nutrients 9(5):E455, doi: 10.3390/nu9050455

Gao Q, Yang M, Zuo Z (2018) Overview of the anti-inflammatory effects, pharmacokinetic properties and clinical efficacies of arctigenin and arctiin from Arctium lappa L. Acta Pharmacologica Sinica 39:787-801

Gao Y, Wei Y, Wang Y, Gao F, Chen Z (2017). Lycium barbarum: A Traditional Chinese Herb and a promising anti-aging agent. Aging and Disease 8:778-791
Garai S, Ghosh R, Bandopadhyay PP, Mandal NC, Chattopadhyay A (2018) Anti-microbial and anti-cancer properties of echinocystic acid extracted from Luffa cylindrica. Journal of Food Processing and Technology 9:717, doi: 10.4172/2157-7110.1000717

Ghasemzadeh A, Jaafar HZE, Baghdadi A, Tayebi-Meigooni A (2018) Formation of 6-, 8and 10-Shogaol in Ginger through application of different drying methods: Altered antioxidant and antimicrobial activity. Molecules 23(7):E1646, doi: 10.3390/molecules23071646

Ghayur MN, Gilani AH, Khan A, Amor EC, Villaseñor IM, Choudhary MI (2006) Presence of calcium antagonist activity explains the use of Syzygium samarangense in diarrhoea. Phytotherapy Research 20:49-52

Ghelani HS, Patel BM, Gokani RH, Rachchh MA (2014) Evaluation of polyherbal formulation (SJT-HT-03) for antihypertensive activity in albino rats. Ayu 35:452-457

Gobinath D, Madhu AN, Prashant G, Srinivasan K, Prapulla SG (2010) Beneficial effect of xylooligosaccharides and fructo-oligosaccharides in streptozotocin-induced diabetic rats. British Journal of Nutrition 104:40-47

Goldberg I (2012) Functional Foods: Designer Foods, Pharmafoods, Nutraceuticals. Springer, New York, USA

Gonçalves RF, Silva AMS, Silva AM, Valentão P, Ferreres F, Gil-Izquierdo A, Silva JB, Santos D, Andrade PB (2013) Influence of taro (Colocasia esculenta L. Shott) growth conditions on the phenolic composition and biological properties. Food Chemistry 141:3480-3485

Grimson A, Ng G, Denardi L (2016) Las organizaciones de inmigrantes chinos en Argentina. Migración y desarrollo 14(26):25-73

Gupta A, Mahajan S, Sharma R (2015) Evaluation of antimicrobial activity of Curcuma longa rhizome extract against Staphylococcus aureus. Biotechnology Reports (Amsterdam) 6:51-55

Gürbüz N, Uluişik S, Frary A, Frary A, Doğanlar S (2018) Health benefits and bioactive compounds of eggplant. Food Chemistry 268:602-610 
Ha H, Lim HS, Lee MY, Shin IS, Jeon WY, Kim $\mathrm{JH}$, Shin HK (2015) Luffa cylindrica suppresses development of Dermatophagoides farinae-induced atopic dermatitis-like skin lesions in $\mathrm{Nc} / \mathrm{Nga}$ mice. Pharmaceutical Biology 53:555-562

Hashiguchi A, Hitachi K, Zhu W, Tian J, Tsuchida K, Komatsu S (2017) Mung bean (Vigna radiata) coat extract modulates macrophage functions to enhance antigen presentation: A proteomic study. Journal of Proteomics 161:2637

He YK, Yao YY, Chang YN (2015) Characterization of anthocyanins in Perilla frutescens var. acuta extract by advanced UPLC-ESI-IT-TOF-MSn method and their anticancer bioactivity. Molecules 20:9155-9169

Hempen CH, Fischer T (2009) A Materia Medica for Chinese Medicine: Plants, Minerals, and Animal Products. Elsevier Health Sciences, Munich, Germany

Hernández C, Ascacio-Valdés J, De la Garza H, Wong-Paz J, Aguilar CN, Martínez-Ávila GC, Castro-López C, Aguilera-Carbó A (2017) Polyphenolic content, in vitro antioxidant activity and chemical composition of extract from Nephelium lappaceum L. (rambutan) husk. Asian Pacific Journal of Tropical Medicine 10:1201-1205

Hlel TB, Belhadj F, Gül F, Altun M, Yağlıoğlu AŞ, Demirtaş I, Marzouki MN (2017) Variations in the bioactive compounds composition and biological activities of Loofah (Luffa cylindrica) fruits in relation to maturation stages. Chemistry and biodiversity 14:10, doi: 10.1002/cbdv.201700178.

Hong EJ, Jung EM, Lee GS, Kim JY, Na KJ, Park MJ, Kang HY, Choi KC, Seong YH, Choi IG, Jeung EB (2010) Protective effects of the pyrolyzates derived from bamboo against neuronal damage and hematoaggregation. Journal ofEthnopharmacology 128: 594-599

Hosseini J, Mamaghani AM, Hosseinifar H, Gilani MAS, Dadkhah F, Sepidarkish M (2016) The influence of ginger (Zingiber officinale) on human sperm quality and DNA fragmentation: A double-blind randomized clinical trial. International Journal of Reproductive BioMedicine (Yazd) 14:533-540
Hsu CC, Huang YC, Yin MC, Lin SJ (2006) Effect of Yam (Dioscorea alata compared to Dioscorea japonica) on gastrointestinal function and antioxidant activity in mice. Journal of Food Science 71:S513-S516

Hu SH, Wang JC, Kung HF, Wang JT, Lee WL, Yang $H$ (2004) Antimicrobial effect of extracts of Cruciferous vegetables. Kaohsiung Journal of Medical Sciences 20:591-598

Hu S.Y (2005) Food plants of China. Chinese University Press, Hong Kong, China

Hu X, Qu Y, Chu Q, Li W, He J (2018) Investigation of the neuroprotective effects of Lycium barbarum water extract in apoptotic cells and Alzheimer's disease mice. Molecular Medicine Reports 17:3599-3606

Hur HJ, Lee AS (2017) Protective effect of Allium tuberosum extract on vascular inflammationin tumor necrosis factor- $\alpha-$ induced Human vascular endothelial cells. Journal of Cancer Prevention 22:228-233

Hurrell JA (2014) Urban Ethnobotany in Argentina: Theoretical advances and methodological strategies. Ethnobiology and Conservation 3:2, doi: 10.15451/ec2014-6-3.3-111

Hurrell JA, Pochettino ML (2014) Urban Ethnobotany: theoretical and methodological contributions. In: Albuquerque UP, Cruz, LVFC, Lucena RFP, Alves RRN (Eds.) Methods and Techniques in Ethnobiology and Ethnoecology. Springer, New York, pp. 293-309

Hurrell JA, Puentes JP (2013) Medicinal and aromatic species of Asteraceae commercialized in the conurbation Buenos Aires-La Plata (Argentina). Ethnobiology and Conservation 2:7, doi 10.15451/ec2013-8-2.7-140

Hurrell JA, Puentes, JP (2017) Plant species and products of the Traditional Chinese Phytotherapy in the Ciudad Autónoma de Buenos Aires, Argentina. Ethnobiology and Conservation 6:1, doi: 10.15451/ec2017026.1143

Hurrell JA, Pochettino ML, Puentes JP, Arenas PM (2013) Del marco tradicional al escenario urbano: Plantas ancestrales devenidas suplementos dietéticos en la conurbación Buenos Aires-La Plata, Argentina. Boletín Latinoamericano y de Caribe de Plantas Medicinales y Aromáticas 12:499-515 
Hurrell JA, Arenas PM, Cristina I (2015a) EI conocimiento botánico en zonas urbanas: potenciadores cognitivos comercializados en el Área Metropolitana de Buenos Aires (Argentina). Gaia Scientia 9:17-31

Hurrell JA, Puentes JP, Arenas PM (2015b) Medicinal plants with cholesterol-lowering effect marketed in the Buenos Aires-La Plata conurbation, Argentina: An Urban Ethnobotany study. Ethnobiology and Conservation 4:6, doi: 10.15451/ec2015-7-4.6-112

Hurrell JA, Morales M, Fortunato RH (2016) Legumbres funcionales $y$ nutracéuticas comercializadas en el Área Metropolitana de Buenos Aires, Argentina. Bonplandia (Corrientes) 25:115-128

Hussain F, Irshad A, Shahid M (2018) Study of selected therapeutic potentials of Momordica charantia fruit. Journal of Biological Regulators and Homeostatic Agents 32:859-862

Hwang JT, Shin EJ, Chung MY, Park JH, Chung S, Choi HK (2018) Ethanol extract of Allium fistulosum inhibits development of nonalcoholic fatty liver disease. Nutrition Research and Practice 12:110-117

Ibrahim SR, Mohamed GA (2015) Litchi chinensis: medicinal uses, phytochemistry, and pharmacology. Journal of Ethnopharmacology 174:492-513

Ibrahim Sayeed VK, Satish S, Kumar A, Hegde K (2017) Pharmacological activities of Vigna unguiculata (L) Walp: A review. International Journal of Pharma and Chemical Research 3:4449

Igarashi M, Miyazaki, Y (2013) A review on bioactivities of Perilla: progress in research on the functions of Perilla as medicine and food. Evidence-Based Complementary and Alternative Medicine 2013:925342, doi: 10.1155/2013/925342

INDEC (2018) Instituto Nacional de Estadística y Censos. Censo 2010 [http://www.indec.gob.ar/] Accessed December 20th 2018

Ivorra MD, Payá M, Villar A (1989) A review of natural products and plants as potential antidiabetic drugs. Journal of Ethnopharmacology 27:243-275
James-Martin G, Williams G, Stonehouse W, O'Callaghan N, Noakes M (2015) Health and nutritional properties of pears (Pyrus): A literature review. CSIRO, Adelaide, Australia

Jayathilake C, Visvanathan $R$, Deen $A$, Bangamuwage R, Jayawardana BC, Nammi S, Liyanage R (2018). Cowpea: an overview on its nutritional facts and health benefits. Journal of the Science of Food and Agriculture 98:47934806

Jeon S, Lee CH, Liu QF, Kim GW, Koo BS, Pak SC (2014) Alteration in brainderived neurotrophic factor (BDNF) after treatment of mice with herbal mixture containing Euphoria Iongana, Houttuynia cordata and Dioscorea japonica. Daru 22:77, doi: 10.1186/s40199-0140077-2

Jeong JT, Moon JH, Park KH, Shin CS (2006) Isolation and characterization of a new compound from Prunus mume fruit that inhibits cancer cells. Journal of Agricultural and Food Chemistry 54:2123-2128

Ji X, Peng Q, Yuan Y, Shen J, Xie X, Wang M (2017) Isolation, structures and bioactivities of the polysaccharides from jujube fruit (Ziziphus jujube Mill.): A review. Food Chemistry 227:349-357

Jia S, Shen M, Zhang F, Xie J (2017) Recent advances in Momordica charantia: Functional components and biological activities. International Journal of Molecular Sciences 18(12):E2555, doi: 10.3390/ijms18122555

Jiang GH, Nam SH, Yim SH, Kim YM, Gwak HJ, Eun JB (2016) Changes in total phenolic and flavonoid content and antioxidative activities during production of juice concentrate from Asian pears (Pyrus pyrifolia Nakai). Food Science and Biotechnology 25(Suppl 1):47-51

Jiang X, Kuang F, Kong F, Yan C (2016) Prediction of the antiglycation activity of polysaccharides from Benincasa hispida using a response surface methodology. Carbohydrate Polymers 151:358-363

Jiang Y, Ng TB, LiuZ,Wang C, Li N, Qiao W, Liua $F$ (2011) Immunoregulatory and anti-HIV-1 enzyme activities of antioxidant components from lotus (Nelumbo nucifera Gaertn.) rhizome. Bioscience Reports 31:381-390 
Jiao D, Yu MC, Hankin JH, Low SH, Chung FL (1998) Total isothiocyanate contents in cooked vegetables frequently consumed in Singapore. Journal of Agricultural and Food Chemistry 46:1055-1058

Jones LD, Pangloli P, Krishnan HB, Dia VP (2018) BG-4, a novel bioactive peptide from Momordica charantia, inhibits lipopolysaccharide-induced inflammation in THP-1 human macrophages. Phytomedicine 42:226-232

Joo HK, Choi S, Lee YR, Lee EO, Park MS, Lim YP, Park JT, Jeon BH (2017) Ethanol extract of Brassica rapa ssp. pekinensis suppresses tumor necrosis factor- $\alpha$-induced inflammatory response in human umbilical vein endothelial cells. Journal of Medicinal Food 20:511-518

Jung HY, Ji Y, Kim NR, Kim DY, Kim KT, Choi BH (2016) A Fomitopsis pinicola Jeseng Formulation has an antiobesity effect and protects against hepatic steatosis in mice with high-fat diet-induced obesity. EvidenceBased Complementary and Alternative Medicine 2016:7312472, doi: 10.1155/2016/7312472

Juritsch AF, Moreau R (2018) Role of soybeanderived bioactive compounds in inflammatory bowel disease. Nutrition Reviews 76:618-638

Kalra EK (2003) Nutraceutical. Definition and introduction. AAPS PharmScience 5:27-28

Kang MJ, Kim JH, Choi HN, Kim MJ, Han JH, Lee JH, Kim JI (2010) Hypoglycemic effects of Welsh onion in an animal model of diabetes mellitus. Nutrition Research and Practice 4:486491

Khan A, Pan JH, Cho S, Lee S, Kim YJ, Park YH (2017) Investigation of the hepatoprotective effect of Prunus mume Sieb. \& Zucc. extract in a mouse model of alcoholic liver injury through high-resolution metabolomics. Journal of Medicinal Food 20:734-743

Khandaker M, Sarwar J, Mat N, Boyce AN (2015) Bioactive constituents, antioxidant and antimicrobial activities of three cultivars of wax apple (Syzygium samarangense L.) fruits. Research Journal Biotechnology 10:7-16

Khayungarnnawee A, Sematong T, Banchonglikitkul C, Thubthimthed S (2018) Cardiovascular effects of the ethanolic extract of Ipomoea aquatica Forssk. in rat. Thai Journal of Pharmaceutical Sciences 42(Suppl):72-75
Kim CJ, Shin SH, Kim BJ, Kim CH, Kim JH, Kang HM, Park BS, Kim IR (2018) The effects of Kaempferol-inhibited autophagy on osteoclast formation. International Journal of Molecular Sciences 19(1):E125, doi: 10.3390/ijms19010125

Kim GN, Shin MR, ShinSH, Lee AR, Lee JY, Seo BI, Kim MY, Kim TH, Noh JS, Rhee MH, Roh SS (2016) Study of antiobesity effect through inhibition of pancreatic lipase activity of Diospyros kaki fruit and Citrus unshiu peel. BioMed Research International 2016:1723042, doi: $10.1155 / 2016 / 1723042$

Kim HH, Kim DS, Kim SW, Lim SH, Kim DK, Shin TY, Kim SH (2013) Inhibitory effects of Diospyros kaki in a model of allergic inflammation: role of cAMP, calcium and nuclear factor-kB. International Journal of Molecular Medicine 32:945-951

Kim HJ, Kang MA, Kim SH, Yim SH, Lee IS (2011) Bioactive phenolic constituents from the culms of Phyllostachys bambusoides. Natural Product Sciences 17:267-272

Kim HS, Ku KM, Suh JK, Kang YH (2009) Quinone reductase inductive activity and growth inhibitory effect against hepatoma cell of oriental melon extract. Journal of BioEnvironment Control 18:448-453

Kim J, Kang H, Choi H, Jo A, Oh DR, Kim Y, Im S, Lee SG, Jeong KI, Ryu GC, Choi C (2018) Aqueous extract of Perilla frutescens var. acuta relaxes the ciliary smooth muscle by increasing NO/cGMP content in vitro and in vivo. Molecules 23(7):E1777, doi: 10.3390/molecules23071777

Kim JH, Suh JK, Kang YH (2012) Anticancer effects of the extracts of oriental melon (Cucumis melo L. var. makuwa Makino) seeds. Korean Journal of Plant Resources 25:647-651

Kim JS, Kim MJ, Park MH, Ryu BM, Moon GS (2008) Angiotensin converting enzyme inhibition and antihypertensive effects of Phyllostachys pubescens culm extracts in spontaneously hypertensive rats. Journal of the Korean Society of Food Science and Nutrition 37:27-34

Kim KH, Moon E, Kim SY, Lee KR (2010) Lignans from the tuber-barks of Colocasia antiquorum var. esculenta and their antimelanogenic activity. Journal of Agricultural and Food Chemistry 58:4779-4785 
Kim MJ, Moon YY, Tou JC, Mou B, Waterland NL (2016) Nutritional value, bioactive compounds and health benefits of lettuce (Lactuca sativa). Journal of Food Composition and Analysis 49:19-34

Kim MS, Bang JH, Lee J, Han JS, Kang HW, Jeon WK (2016) Fructus mume ethanol extract prevents inflammation and normalizes the Septohippocampal Cholinergic System in a rat model of Chronic Cerebral Hypoperfusion. Journal of Medicinal Food 19:196-204

Kim S, Park SH, Lee HN, Park T (2008) Prunus mume extract ameliorates exercise-induced fatigue in trained rats. Journal of Medicinal Food 11:460-468

Kocevski D, Du M, Kan J, Jing C, Lačanin I, Pavlović H (2013) Antifungal effect o Allium tuberosum, Cinnamomum cassia, and Pogostemon cablin essential oils and their components against population of Aspergillus species. Journal of Food Science 78:M731-M737

Komatsu KI, Takanari J, Maeda T, Kitadate K, Sato T, Mihara Y, Uehara K, Wakame K (2016) Perilla leaf extract prevents atopic dermatitis induced by an extract of Dermatophagoides farinae in NC/Nga mice. Asian Pacific Journal of Allergy and Immunology 34:272-277

Kono R, Nakamura M, Nomura S, Kitano N, Kagiya $\mathrm{T}$, Okuno $\mathrm{Y}$, Inada $\mathrm{KI}$, Tokuda $\mathrm{A}$, Utsunomiya $H$, Ueno M (2018) Biological and epidemiological evidence of anti-allergic effects of traditional Japanese food ume (Prunus mume). Scientific Reports 8(1):11638, doi: $10.1038 / s 41598-018-30086-5$

Kooti W, Daraei N (2017) A review of the antioxidant activity of Celery (Apium graveolens L.). Journal of Evidence-Based Complementary and Alternative Medicine 22:1029-1034

Koyyati R, Kudle KR, Padigya PMR (2016) Evaluation of antibacterial and cytotoxic activity of green synthesized cobalt nanoparticles using Raphanus sativus var. Iongipinnatus leaf extract. International Journal of PharmTech Research 9:466-472

Krishnapriya TV, Suganthi A (2017) Biochemical and phytochemical analysis of Colocasia esculenta (L.) Schott tubers. International Journal of Research in Pharmacy and Pharmaceutical Sciences 2:21-25
Kucekova Z, Mlcek J, Humpolicek P, Rop O, Valasek P, Saha P (2011) Phenolic compounds from Allium schoenoprasum, Tragopogon pratensis and Rumex acetosa and their antiproliferative effects. Molecules 16:92079217

Kuroda R, Kazumura K, Ushikata M, Minami Y, Kajiya K (2018) Elucidating the improvement in vascular endothelial function from Sakurajima Daikon and its mechanism of action: A Comparative study with Raphanus sativus. Journal of Agricultural and Food Chemistry 66:8714-8721

Kumar S, Sharma G, Sharma A, George M, Lincey $J$ (2011) Anticonvulsant activity of chloroform extract of Phyllostachys bambusoides. International Journal of Pharmacy and Pharmaceutical Sciences 3:125127

Kumar S, Chakravarthi S, Chiew GS, Subramaniam T, Palanisamy U, Radhakrishnan A, Haleagrahara N (2012) Protective effects of Nephelium lappaceum rind extract against collagen-induced arthritis in dark agouti rats. Journal of Biological Sciences 12:385-392

Kumaran A, Ho CC, Hwang LS (2018) Protective effect of Nelumbo nucifera extracts on beta amyloid protein induced apoptosis in PC12 cells, in vitro model of Alzheimer's disease. Journal of Food and Drug Analysis 26:172-181

Kunworarath N, Rangkadilok N, Suriyo T, Thiantanawat A, Satayavivad J (2016) Longan (Dimocarpus longan Lour.) inhibits lipopolysaccharide-stimulated nitric oxide production in macrophages by suppressing NF-KB and AP-1 signaling pathways. Journal of Ethnopharmacology 179:156-161

Kundu N, Campbell P, Hampton B, Lin CY, Ma X, Ambulos N, Zhao XF, Goloubeva O, Holt D, Fulton AM (2012) Antimetastatic activity isolated from Colocasia esculenta (taro). Anticancer Drugs 23:200-211

Kusano $\mathrm{Y}$, TsujiharaN, Masui $\mathrm{H}$, Kozai $\mathrm{H}$, Takeuchi W (2016) Consumption of Japanese Yam improves lipid metabolism in highcholesterol diet-fed rats. Journal of Nutritional Science and Vitaminology (Tokyo) 62:350-360

Kwak Y, Lee J, Ju J (2016) Anti-cancer activities of Brassica juncea leaves in vitro. EXCLI Journal 15:699-710 
La GX, Yang TG, Fang P, Guo HX, Hao X, Huang SM (2013) Effect of NH4+/NO3- ratios on the growth and bolting stem glucosinolate content of Chinese kale (Brassica alboglabra L.H. Bailey). Australian Journal of Crop Science 7:618-624

Ladio AH, Albuquerque UP (2016) Urban Ethnobiology. In: Albuquerque UP, Alves RRN (Eds.) Introduction to Ethnobiology. Springer, New York, pp. 33-38

Lee AY, Hwang BR, Lee MH, Lee S, Cho EJ (2016a) Perilla frutescens var. japonica and rosmarinic acid improve amyloid- $\beta 25-35$ induced impairment of cognition and memory function. Nutrition Research and Practice $10: 274-281$

Lee AY, Wu T, Hwang BR, Lee J, Lee MH, Lee S, Cho EJ (2016b) The neuro-protective effect of the methanolic extract of Perilla frutescens var. japonica and rosmarinic acid against $\mathrm{H}_{2} \mathrm{O}_{2}$-induced oxidative stress in $\mathrm{C} 6$ glial cells. Biomolecules and therapeutics (Seoul) 24:338-345

Lee JB, Miyake S, Umetsu R, Hayashi K, Chijimatsu T, Hayashi T (2012) Anti-influenza A virus effects of fructan from Welsh onion (Allium fistulosum L.). Food Chemistry 134:2164-2168

Lee JJ, Kim HA, Lee J (2018) The effects of Brassica juncea L. leaf extract on obesity and lipid profiles of rats fed a high-fat/highcholesterol diet. Nutrition Research and Practice 12:298-306

Lee JH, Yang HS, Park KW, Kim JY, Lee MK, Jeong IY, Shim KH, Kim YS, Yamada K, Seo KI (2009) Mechanisms of thiosulfinates from Allium tuberosum L.induced apoptosis in HT29 human colon cancer cells. Toxicology Letters 188:142-147

Lee JK, Choi EH, Lee KG, Chun HS (2005) Alleviation of aflatoxin B1-induced oxidative stress in HepG2 cells by volatile extract from Allii Fistulosi Bulbus. Life Sciences 77:28962910

Lee KA, Jin HY, Baek HS, Park TS (2013) The protective effects of DA-9801 (Dioscorea extract) on the peripheral nerves in streptozotocin-induced diabetic rats. Journal of Nutritional Science and Vitaminology (Tokyo) 59:437-446
Lee NK, Lee JH, LimSM, Lee KA, Kim YB, Chang PS, Paik HD (2014) Antiviral activity of subcritical water extract of Brassica juncea against influenza virus $A / H 1 N 1$ in nonfat milk. Journal of Dairy Science 97:5383-5386

Lee SW, Kim SJ, Kim H, Yang D, Kim HJ, Kim BJ (2017) Effects of Prunus mume Siebold \& Zucc. in the pacemaking activity of interstitial cells of Cajal in murine small intestine. Experimental and Therapeutic Medicine 13:327334

Leivas CL, lacomini M, Cordeiro LM (2016) Substituted galacturonan from starfruit: Chemical structure and antinociceptive and anti-inflammatory effects. International Journal of Biological Macromolecules 84:295-300

Li XM, Miao Y, Su QY, YaoC, Li HH, Zhang GM (2016) Gastroprotective effects of arctigenin of Arctium lappa L. on a rat model of gastric ulcers. Biomedical Reports 5:589-594

Lim TK (2012a) Edible Medicinal and Non Medicinal Plants. 3, Fruits. Springer, Dordrecht, Nederland

Lim TK (2012b) Edible Medicinal and Non Medicinal Plants. 4, Fruits. Springer, Dordrecht, Nederland

Lim TK (2015) Edible Medicinal and Non Medicinal Plants. 9, Modified Stems, Roots, Bulbs. Springer, Dordrecht, Nederland

Lin PL, Lin KW, Weng CF, Lin KC (2009) Yam storage protein dioscorins from Dioscorea alata and Dioscorea japonica exhibit distinct immunomodulatory activities in mice. Journal of Agricultural and Food Chemistry 57:4606-4613

Liu J (2011) Chinese Food. Cambridge University Press, Cambridge, UK

Liu R, Zheng Y, Cai Z, Xu B (2017) Saponins and flavonoids from Adzuki Bean (Vigna angularis) ameliorate high-fat diet-induced obesity in ICR Mice. Frontiers in Pharmacology 8:687, doi: 10.3389/fphar.2017.00687

Liu W, Wang J, Zhang Z, Xu J, Xie Z, Slavin M, Gao X (2014) In vitro and in vivo antioxidant activity of a fructan from the roots of sArctium lappa L. International Journal of Biological Macromolecules 65:446-453

Liu XC, Zhou L, Liu Q, Liu ZL (2015) Laboratory evaluation of larvicidal activity of the essential oil of Allium tuberosum roots and its selected major constituent compounds against Aedes albopictus (Diptera: Culicidae). Journal of Medical Entomology 52:437-441 
Liu Y, Wang Z, Zhang J (2015a) Dietary Chinese Herbs: Chemistry, Pharmacology and Clinical Evidence. Springer, Wien, Österreich

Liu Y, Wang G, Yang M,Chen $\mathrm{H}$, Zhao Y, Yang S, Sun C (2015b) Arctigenin reduces blood pressure by modulation of nitric oxide synthase and NADPH oxidase expression in spontaneously hypertensive rats. Biochemical and Biophysical Research Communications 468:837-842

Liu YT, Perera CO, Suresh V (2007) Comparison of three chosen vegetables with others from South East Asia for their lutein and zeaxanthin content. Food Chemistry 101:1533-1539

Lopes LAR, Martins MDCCE, Farias LM, Brito AKDS, Lima GM, Carvalho VBL, Pereira CFC, Conde Júnior AM, Saldanha T, Arêas JAG, Silva KJDE, Frota KMG (2018) Cholesterol-lowering and Liver-protective effects of cooked and germinated Mung beans (Vigna radiata). Nutrients 10(7):E821, doi: 10.3390/nu10070821

Lou SN, Ho CT (2017) Phenolic compounds and biological activities of small-size Citrus: kumquat and calamondin. Journal of Food and Drug Analysis 25:162-175

Lu X, Mo X, Guo H, Zhang Y (2012) Sulfation modification and anticoagulant activity of the polysaccharides obtained from persimmon (Diospyros kaki L.) fruits. International Journal of Biological Macromolecules 51:1189-1195

Lust J (2014) The Herb Book. Dover Publications, New York, USA

Ma Y, Ma H, Eun JS, Nam SY, Kim YB, Hong JT, Lee MK, Oh KW (2009) Methanol extract of Longanae Arillus augments pentobarbitalinduced sleep behaviors through the modification of GABAergic systems. Journal of Ethnopharmacology 122:245-250

Maghsoumi-Norouzabad L, Alipoor B, Abed R, Eftekhar Sadat B, Mesgari-Abbasi M, Asghari Jafarabadi M (2016) Effects of Arctium lappa L. (Burdock) root tea on inflammatory status and oxidative stress in patients with knee osteoarthritis. International Journal of Rheumatic Diseases 19:255-261

Mahajan RT, Chopda MZ (2009) PhytoPharmacology of Ziziphus jujuba Mill. A plant review. Pharmacognosy Reviews 3:320-329
Malakar C, Choudhury PPN (2015) Pharmaclogical potentiality and medicinal uses of Ipomoea aquatica. A review. Asian Journal of Pharmaceutical and Clinical Research 8:60-63

Man S, Ma J, Yao J, Cui J, Wang C, Li Y, Ma L, Lu F (2017) Systemic perturbations of key metabolites in type 2 diabetic rats treated by polyphenol extracts from Litchi chinensis seeds. Journal of Agricultural and Food Chemistry 65:7698-7704

Manasa S, Raju AB (2014) Anti-amnesic activity of Solanum melongenaL extract. Postepy Fitoterpii 1:1-7.

Manvar MN, Desai TR (2013) Phytochemical and pharmacological profile of Ipomoea aquatica. Indian Journal of Medical Sciences 67:49-60

Martin GJ (1995) Ethnobotany. A methods manual. Chapman and Hall, London, UK

Marzocco S, Calabrone L, Adesso S, Larocca M, Franceschelli S, Autore G, Martelli G, Rossano R (2015) Anti-inflammatory activity of horseradish (Armoracia rusticana) root extracts in LPS-stimulated macrophages. Food and Function 6:3778-3788

Matsumura Y, Ito T, Yano H, Kita E, Mikasa K, Okada M, Furutani A, Murono $Y$, Shibata $M$, Nishii Y, Kayano S (2016) Antioxidant potential in non-extractable fractions of dried persimmon (Diospyros kaki). Food Chemistry 202:99-103

Maxwell T, Chun SY, Lee KS, Kim S, Nam KS (2017) The anti-metastatic effects of the phytoestrogen arctigenin on human breast cancer cell lines regardless of the status of ER expression. International Journal of Oncology 50:727-735

Medeiros PM, Soldati GT, Alencar NL, Vandebroek I, Pieroni A, Hanazaki N, Albuquerque UP (2012) The Use of Medicinal Plants by Migrant People: Adaptation, Maintenance, and Replacement. EvidenceBased Complementary and Alternative Medicine 2012: 807452, doi:10.1155/2012/807452.

Meira M, Silva EP, David JM, Juceni PD (2012) Review of the genus Ipomoea: traditional uses, chemistry and biological activities. Revista Brasileira de Farmacognosia 22:682-713 
Meng FY, Ning YL, Qi J, He Z, Jie J, Lin JJ, Huang YJ, Li FS, Li XH (2014) Structure and antitumor and immunomodulatory activities of a water-soluble polysaccharide from Dimocarpus Iongan pulp. International Journal of Molecular Sciences 15:5140-5162

Mesaik AM, Poh HW, Bin OY, Elawad I, Alsayed $B$ (2018) In vivo anti-inflammatory, antibacterial and anti-diarrhoeal activity of Ziziphus Jujuba fruit extract. Open Access Macedonian Journal of Medical Sciences 6:757766

Miraj S (2016) Pharmacological effects of Saccharum officinarum L. Der Pharmacia Lettre 8:223-225

Mitani T, Ota K, Inaba N, Kishida K, Koyama HA (2018) Antimicrobial activity of the phenolic compounds of Prunus mume against Enterobacteria. Biological and Pharmaceutical Bulletin 41:208-212

Mnayer D, Fabiano-Tixier AS, Petitcolas E, Hamieh T, Nehme N, Ferrant C, Fernández X, Chemat $F$ (2014) Chemical composition, antibacterial and antioxidant activities of six essentials oils from the Alliaceae family. Molecules 19:20034-20053

Mohamad S, Ismail NN, Parumasivam T, Ibrahim P, Osman H, A Wahab H (2018) Antituberculosis activity, phytochemical identification of Costus speciosus (J. Koenig) Sm., Cymbopogon citratus (DC.) Stapf., and Tabernaemontana coronaria (L.) Willd. and their effects on the growth kinetics and cellular integrity of Mycobacterium tuberculosis H37Rv. BMC Complementary and Alternative Medicine 18(1):5, doi: 10.1186/s12906-017-2077-5

Monteiro JM, Lima Araujo E, Cavalcanti Amorim EL, Albuquerque UP (2010) Local markets and medicinal plant commerce: A review with emphasis on Brazil. Economic Botany 64(4):352-366

Morita A, Tai A, Ito H, Ganeko N, Aizawa S (2016) Proanthocyanidins in an astringent persimmon inhibit Salmonella pathogenicityisland 1 (SPI1) secretion. Journal of the Science of Food and Agriculture 96:17981802

Mushtaq A, Naqvi SF, Anwar R, Jamil M, Anwar $H$, Bashir A,ul-Ain Q, Ayesha B (2016) Evaluation of hypolipidemic activity of Allium schoenoprasum in albino rats. British Journal of Pharmaceutical Research 14:1-10
Muthu N, Lee SY, Phua KK, Bhore SJ (2016) Nutritional, medicinal and toxicological attributes of Star-Fruit (Averrhoa carambola L.): A review. Bioinformation 12:420-424

Nahata A, Dixit VK (2014) Evaluation of $5 \alpha-$ reductase inhibitory activity of certain herbs useful as antiandrogens. Andrologia 46:592601

Naidu BB, Palaksha MN, Ravishankar K, Naveen M, Basha N (2014) Evaluation of antidiarrhoeal and antiulcer activities of hydroalcoholic extract of Luffa aegyptiaca fruits. Research Journal of Pharmacology and Pharmacodynamics 6:184-189

Nandecha C, Nahata A, Dixit VK (2010) Effect of Benincasa hispida fruits on testosterone induced prostatic hypertrophy in albino rats. Current Therapeutic Research 71:331-343

Nethaji R, Thooyavan G, Mullai Nilla K, Ashok K (2015) Phytochemical profiling, antioxidant and antimicrobial activity of methanol extract in Rambutan fruit (Nephelium lappaceum) epicarp against the human pathogens. International Journal of Current Innovation Research 1:201-206

Newman JM (2004) Food Culture in China. Greenwood Press, Westport, USA

Ng YM, Yang Y, Sze KH, Zhang X, Zheng YT, Shaw PC (2011) Structural characterization and anti-HIV-1 activities of arginine/glutamate-rich polypeptide Luffin P1 from the seeds of sponge gourd (Luffa cylindrica). Journal of Structural Biology 174:164-172

Nguyen NM, Gonda S, Vasas G (2013) A review on the phytochemical composition and potential medicinal uses of Horseradish (Armoracia rusticana) root. Food Reviews International 29:261-215

Noorafshan A, Ashkani-Esfahani S (2013) A review of therapeutic effects of Curcumin. Current Pharmaceutical Design 19:2032-2046

Nwaka AC, Okeyikam CC, Bayim PR, Okafor IN (2014) Effect of Shaddock Citrus fruit juice on the lipid profile, fasting blood glucose level and kidney function of male Wistar albino rats. Journal of Pharmacy and Biological Sciences 9:71-74 
Oguro Y, Yamazaki H, Takagi M, Takaku H (2014) Antifungal activity of plant defensin AFP1 in Brassica juncea involves the recognition of the methyl residue in glucosylceramide of target pathogen Candida albicans. Current Genetics 60:89-97

Okada Y, Okada M (2015) Effects of methanolic extracts from edible plants on endogenous secretory receptor for advanced glycation end products induced by the high glucose incubation in human endothelial cells. Journal of Pharmacy and Bioallied Sciences 7:145-150

Okuyama E, Ebihara H, Takeuchi H, Yamazaki M (1999) Adenosine, the anxiolytic-like principle of the arillus of Euphoria Iongana. Planta Medica 65:115-119

Pallavi R, Elakkiya S, Tennety SSR, Suganya Devi P (2012) Anthocyanin analysis and its anticancer property from sugarcane (Saccharum officinarum L.) peel. International Journal of Research in Pharmaceutical Sciences 2:338-345

Panee J (2015) Potential medicinal application and toxicity evaluation of extracts from Bamboo plants. Journal of Medicinal Plants Research 9:681-692

Pang X, Panee J (2016) Anti-inflammatory function of Phyllostachys edulis extract in the hippocampus of HIV-1 transgenic rats. Journal of HIV and AIDS 2(3), doi: 10.16966/23805536.126

Park C, Jin CY, Kim GY, Jeong YK, Kim WJ, Choi $\mathrm{YH}$ (2011) Induction of apoptosis by ethanol extract of Prunus mumein U937 human leukemia cells through activation of caspases. Oncology Reports 26:987-993

Park HR, Lee HS, Cho SY, Kim YS, Shin KS (2013) Anti-metastatic effect of polysaccharide isolated from Colocasia esculenta is exerted through immunostimulation. International Journal of Molecular Medicine 31:361-368

Park KW, Kim SY, Jeong IY, Byun MW, Park KH, Yamada K, Seo KI (2007) Cytotoxic and antitumor activities of thiosulfinates from Allium tuberosum L. Journal of Agricultural and Food Chemistry 55:7957-7961
Park MJ, Song JH, Shon MS, Kim HO, Kwon OJ, Roh SS, Kim CY, Kim GN (2016) Antiadipogenic effects of ethanol extracts prepared from selected medicinal herbs in 3T3-L1 cells. Preventive Nutrition and Food Science 21:227-235

Park S, Kim JH, Son Y, Goh SH, Oh S (2016) Longan (Dimocarpus longan Lour.) fruit extract stimulates osteoblast differentiation via Erk1/2-Dependent RUNX2 Activation. Journal of Microbiology and Biotechnology 26:1063-1066

Park SB, Park GH, Song HM, Son HJ, Um Y, Kim HS, Jeong JB (2017) Anticancer activity of calyx of Diospyros kaki Thunb. through downregulation of cyclin D1 via inducing proteasomal degradation and transcriptional inhibition in human colorectal cancer cells. BMC Complementary and Alternative Medicine 17:445, doi: 10.1186/s12906-017-1954-2.

Park SH, Sung YY, Nho KJ, Kim HK (2015) Protective activity ethanol extract of the fruits of Illicium verum against atherogenesis in apolipoprotein E knockout mice. BMC Complementary and Alternative Medicine 15:232, doi: 10.1186/s12906-015-0750-0

Park SJ, Park DH, Kim DH, Lee S, Yoon BH, Jung WY, Lee KT, Cheong JH, Ryu JH (2010) The memory-enhancing effects of Euphoria longan fruit extract in mice. Journal of Ethnopharmacology 128:160-165

Park SY, Kim HS, Hong SS, Sul D, Hwang KW, Lee D (2009) The neuroprotective effects of traditional oriental herbal medicines against $\beta$-amyloid-induced toxicity. Pharmaceutical Biology 47:976-981

Partap S, Kumar A, Sharma NK, Jha KK (2012) Luffa cylindrica: an important medicinal plant. Journal of Natural Product and Plant Resources 2:127-134

Parvu AE, Parvu M, Vlase L, Miclea P, Mot AC, Silaghi-Dumitrescu R (2014) Anti-inflammatory effects of Allium schoenoprasum L. leaves. Journal of Physiology and Pharmacology 65:309315

Paudel KR, Panth N (2015) Phytochemical profile and biological activity of Nelumbo nucifera. Evidence-Based Complementary and Alternative Medicine 2015:789124, http://dx.doi.org/10.1155/2015/789124 
Pawarn HA, Choudhary PD, Kamat SR (2018) An overview of traditionally used herb, Colocasia esculenta, as a phytomedicine. Medicinal and Aromatic Plants 7:2, doi: 10.4172/2167-0412.1000317

Pereira JV, Bergamo DC, Pereira JO, França Sde C, Pietro RC, Silva-Sousa YT (2005) Antimicrobial activity of Arctium lappa constituents against microorganisms commonly found in endodontic infections. Brazilian Dental Journal 16:192-196

Pereira PR, Silva JT, Verícimo MA, Paschoalin VMF, Teixeira GAPB (2015) Crude extract from taro (Colocasia esculenta) as a natural source of bioactive proteins able to stimulate haematopoietic cells in two murine models. Journal of Functional Foods 18:333-343

Pham HT, Huang W, HanC, Li J, Xie Q, Wei J, Xu X, Lai Z, Huang X, Huang R, Wen Q (2017) Effects of Averrhoa carambola L. (Oxalidaceae) juice mediated on hyperglycemia, hyperlipidemia, and its influence on regulatory protein expression in the injured kidneys of streptozotocin-induced diabetic mice. American Journal of Translational Research 9:36-49

Pieroni A, Price L (2006) Eating and Healing: Traditional Food as Medicine. Hawthorn, New York

Pieroni A, Vandebroek I (2009) Traveling cultures and plants. The ethnobiology and ethnopharmacy of migrations. Berghahn Books, New York, USA

Pieroni A, Muenz H, Akbulut $M$, Başer $K$, Durmuşkahya C (2005) Traditional phytotherapy and trans-cultural pharmacy among Turkish migrants living in Cologne, Germany. Journal of Ethnopharmacology 102:69-88

Pochettino ML, Martínez MR, Itten B, Zucaro M (1997) El uso de plantas medicinales en la atención primaria de la salud: estudio etnobotánico en una población urbana (La Plata, Buenos Aires, Argentina). Parodiana 10:141-152

Pochettino ML, Arenas PM, Sánchez D, Correa R (2008) Conocimiento botánico tradicional, circulación comercial y consumo de plantas medicinales en un área urbana de Argentina. Boletín Latinoamericano y del Caribe de Plantas Medicinales y Aromáticas 7(3):141-148
Pochettino ML, Puentes JP, Buet Costantino F, Arenas PM, Ulibarri EA, Hurrell JA (2012) Functional Foods and Nutraceuticals in a Market of Bolivian Inmigrants in Buenos Aires (Argentina). Evidence-Based Complementary and Alternative Medicine 2012, doi: 10.1155/2012/320193.

Porterfield WM (1951) The principal Chinese vegetable foods and food plants of Chinatown markets. Economic Botany 5:3-37

PubMed (2018) US National Library of Medicine National Institutes of Health. NCBI, National Center for Biotechnology Information [https://www.ncbi.nlm.nih.gov/pubmed] Accessed October 1st 2018

Puentes JP (2016) Plantas medicinales y productos derivados comercializados como antidiabéticos en la conurbación Buenos Aires-La Plata, Argentina. Boletín Latinoamericano y del Caribe de Plantas Medicinales y Aromáticas 15:373-397

Puentes JP (2017) Etnobotánica urbana: el conocimiento botánico local sobre las plantas alimenticias y medicinales, y sus usos, en la conurbación Buenos Aires-La Plata. Tesis Doctoral. Universidad Nacional de La Plata, La Plata, Argentina

Puentes JP, Hurrell JA (2015) Plantas andinas y sus productos comercializados con fines medicinales y alimentarios en el Área Metropolitana Buenos Aires-La Plata, Argentina. Boletín Latinoamericano y del Caribe de Plantas Medicinales y Aromáticas 14:206-236

Qiu X, Jia J (2014) Research advances on TCM anti-tumor effects and the molecular mechanisms. Journal of Cancer Research and Therapeutics 10:8-13

Quinlan M (2005) Considerations for collecting freelists in the field: examples from Ethnobotany. Field Methods 17:1-16

Rabelo TK, Zeidán-Chuliá F, Caregnato FF, Schnorr CE, Gasparotto J, Serafini MR, de Souza Araújo AA, Quintans-Junior LJ, Moreira JCF, Gelain DP (2015) In vitro neuroprotective effect of shikimic acid against hydrogen peroxide-induced oxidative stress. Journal of Molecular Neuroscience 56(4):956-965 
Rahman MS, Jahan N, Rahman SMA, Rashid MA (2015) Analgesic and antidepressant activities of Brassica rapa subspecies chinensis on Swiss-albino mice model. Bangladesh Medical Research Council Bulletin 41:114-120

Rahmatullah M, Shefa TF, Hasan L, Hossain T, Ahmed S, Al Mamun A, Islam R, Rahman S, Chowdhury MH (2010) A study on antinociceptive and anti-hyperglycemic activity of methanol extract of Brassica juncea leaves in mice. Advances in Natural and Applied Sciences 4:221-225

Rashid AM., Lu K, Yip YM, Zhang D (2016) Averrhoa carambola L. peel extract suppresses adipocyte differentiation in 3T3L1 cells. Food and Function 7:881-892

Rastogi S, Pandey MM, Rawat AKS (2017) Spices: Therapeutic potential in cardiovascular health. Current Pharmaceutical Design 23:989-998

Reiff M, O'Connor B, Kronenberg F, Balick MJ, LohrP, Fugh-Berman A, Johnson, KD (2003) Ethnomedicine in urban environment: Dominican healers in New York city. Human Organization 62:12-26

Ribeiro GG, Pessôa LR, Abreu MDC, Corrêa LBNS, D'Avila Pereira A, Chagas MA, Brandão FZ, Costa CAS, Boaventura GT (2018) Taro flour (Colocasia esculenta) increases testosterone levels and gametogenic epithelium of Wistar rats. Journal of Developmental Origins of Health and Disease 9:373-376

Ripa FA, Morshed MT, Al-Sharmin A, Papon SB, Islam MR, Sheikh Z (2014) Central Nervous System depressant, analgesic and antidiarrheal effects of the seed extracts of Dimocarpus Iongan Lour. in rats. Tropical Journal of Pharmaceutical Research 13:235-242

Ritter AM, Ames FQ, Otani F, de Oliveira RM, Cuman R, Bersani-Amado CA (2014) Effects of anethole in nociception experimental models. Evidence-Based Complementary and Alternative Medicine 2014:345829,

doi:

$10.1155 / 2014 / 345829$

Rochfort SJ, Imsic M, Jones R, Trenerry VC, Tomkins B (2006) Characterization of flavonol conjugates in immature leaves of Pak Choi [Brassica rapa ssp. chinensis] by HPLC-DAD and LC-MS/MS. Journal of Agricultural and Food Chemistry 54:4855-4860
Roghani M, Khalili M, Baluchnejadmojarad T, Aghaie M, Ansari F, Sharayeli M (2010) Effect of oral feeding of Allium schoenoprasum $L$. on blood glucose and lipid level in diabetic rats. Journal of Gorgan University of Medical Sciences 12:9-14

Roghani M, Baluchnejadmojarad T, Kord M (2011) Antinociceptive effect of Allium schoenoprasum $L$. oral feeding in diabetic rats. Planta Medica 77:PM109, doi: 10.1055/s0031-1282867.

Sabale V, Kunjwani H, Sabale P (2011) Formulation and in vitro evaluation of the topical antiageing preparation of the fruit of Benincasa hispida. Journal of Ayurveda and Integrative Medicine 2:124-128

Saghir S, Sadikun A, Khaw KY, Murugaiyah V (2013) Star Fruit (Averrhoa carambola L.): From traditional uses to pharmacological activities. Boletín Latinoamericano y del Caribe de Plantas Medicinales y Aromáticas 12:209-219 Principio del formulario

Saghir S, Sadikun A, Al-Suede FS, Majid AM, Murugaiyah V (2016) Antihyperlipidemic, antioxidant and cytotoxic activities of methanolic and aqueous extracts of different parts of Star Fruit. Current Pharmaceutical Biotechnology 17:915-925

Saleh MM, Zwaving JH, Malingré TM, Bos R (1985) The essential oil of Apium graveolens var. secalinum and its cercaricidal activity. Pharmaceutisch Weekblad Scientific Edition 7:277-279

Sandhu DS, Heinrich M (2005) Use of health foods, spices and other botanicals in the Sikh community in London. Phytotherapy Research 19(7):633-642

Sanjaya Kumar YR, Acharya MV (2016) Genus Luffa. An ethnopharmacological and phytochemical review. International Journal of Pharmaceutical Sciences and Research 7:240244

Sassone S, Mera C (2007) Barrios de migrantes en Buenos Aires. Identidad, cultura, cohesión socioterritorial. CEISAL, Bruxelles, Belgium

Sato S, Kataoka S, Kimura A, Mukai Y (2016) Azuki bean (Vigna angularis) extract reduces oxidative stress and stimulates autophagy in the kidneys of streptozotocin-induced early diabetic rats. Canadian Journal of Physiology and Pharmacology 94:1298-1303 
Sawant TP, Panhekar D (2017) A brief review on recent advances of Citrus maxima (chakota). International Journal of Recent Scientific Research 8:19400-19416

Semaming Y, Pannengpetch P, Chattipakorn SC, Chattipakorn N (2015) Pharmacological properties of protocatechuic acid and its potential roles as complementary medicine. Evidence-Based Complementary and Alternative Medicine

http://dx.doi.org/10.1155/2015/593902

2015:593902

Seong GU, Hwang IW, Chung SK (2016) Antioxidant capacities and polyphenolics of Chinese cabbage (Brassica rapa ssp. pekinensis) leaves. Food Chemistry 199:612 618

Sharma D, Rawat I, Goel HC (2015) Anticancer and anti-inflammatory activities of some dietary cucurbits. Indian Journal of Experimental Biology 53:216-221

Shen SC, Chang WC (2013) Hypotriglyceridemic and hypoglycemic effects of vescalagin from Pink wax apple [Syzygium samarangense (Blume) Merrill \& Perry cv. Pink] in high-fructose diet-induced diabetic rats. Food Chemistry 136:858-863

Shen SC, Chang WC, Chang CL (2013) An extract from wax apple (Syzygium samarangense (Blume) Merrill \& Perry) effects glycogenesis and glycolysis pathways in tumor necrosis factor- $\alpha$-treated FL83B mouse hepatocytes. Nutrients 5:455-467

Sheu SY, Fu YT, Huang WD, Chen YA, Lei YC, Yao CH, Hsu FL, Kuo TF (2016) Evaluation of xanthine oxidase inhibitory potential and in vivo hypouricemic activity of Dimocarpus longan Lour. extracts. Pharmacognosy Magazine 12 (Suppl 2):S206-S212

Shi GJ Zheng J, Wu J, Qiao HQ, Chang Q, Niu Y, Sun T, Li YX, Yu JQ (2017) Protective effects of Lycium barbarum polysaccharide on male sexual dysfunction and fertility impairments by activating hypothalamic pituitary gonadal axis in streptozotocin-induced type-1 diabetic male mice. Endocrine Journal 64:907-922

Shi J, Shahidi F, Ho CT (2005) Asian Functional Foods. CRC Press, Boca Raton, USA
Shi J, Liu X, Li Z, Zheng Y, Zhang Q, Liu X (2015) Laboratory evaluation of acute toxicity of the essential oil of Allium tuberosum leaves and its selected major constituents against Apolygus lucorum (Hemiptera: Miridae). Journal of Insect Science 15: doi: 10.1093/jisesa/iev091

Shin EJ, Hur HJ, Sung MJ, Park JH, Yang HJ, Kim MS, Kwon DY, Hwang JT (2013) Ethanol extract of the Prunus mume fruits stimulates glucose uptake by regulating PPAR-y in C2C12 myotubes and ameliorates glucose intolerance and fat accumulation in mice fed a high-fat diet. Food Chemistry 141:4115-4121

Simirgiotis MJ, Adachi $S$, To $S$, Yang $H$, Reynertson KA, Basile MJ, Gil RR, Weinstein IB, Kennelly EJ (2008) Cytotoxic chalcones and antioxidants from the fruits of a Syzygium samarangense (Wax Jambu). Food Chemistry 107:813-819

Simoons FJ (2014) Food in China: A Cultural and Historical Inquiry. CRC Press, Boca Raton, USA

Simsek S, Nehir El S (2015) In vitro starch digestibility, estimated glycemic index and antioxidant potential of taro (Colocasia esculenta) corm. Food Chemistry 168:257-261

Singh A, Navneet (2017) Citrus maxima (Burm.) Merr. A traditional medicine: its antimicrobial potential and pharmacological update for commercial exploitation in herbal drugs. A review. International Journal of ChemTech Research 10:642-651

Singh R, Sharma J, Goyal PK (2014) Prophylactic role of Aerrhoa caranbed a (Star Fruit) extract against chemically induced hepatocellular carcinoma in Swiss Albino Mice. Advances in Pharmacological Sciences 2014:158936, doi: 10.1155/2014/158936

Singh R, Nawale L, Sarkar D, Suresh CG (2016) Two chitotriose-specific lectins show antiangiogenesis, induces caspase-9-mediated apoptosis and early arrest of pancreatic tumor cell cycle. Plos One 11(1):e0146110, doi: 10.1371/journal.pone.0146110

Singh V, Chauhan G, Krishan P, Shri R (2018a) Allium schoenoprasum $L$.: A review of phytochemistry, pharmacology and future directions. Natural Product Research 32:22022216 
Singh V, Krishan P, Shri R (2018b) Antioxidantmediated neuroprotection by Allium schoenoprasum $L$. leaf extract against ischemia reperfusion-induced cerebral injury in mice. Journal of Basic and Clinical Physiology and Pharmacology 29:403-410

Singhal P, Bal LM, Satya S, Sudhakar P, Naik SN (2013) Bamboo shoots: A novel source of nutrition and medicine. Food Science and Nutrition 53:517-534

Sohn HY, Kum EJ, Ryu HY, Jeon SJ, Kim NS, Son KH (2006) Antifungal activity of fistulosides, steroidal saponins, from Allium fistulosum L. Journal of Life Sciences 16:310314

Son ES, Kim YO, Park CG, Park KH, Jeong SH, Park JW, Kim SH (2017) Coix lacryma-jobi var. mayuen Stapf sprout extract has antimetastatic activity in colon cancer cells in vitro. BMC Complementary and Alternative Medicine 17(1):486, doi: 10.1186/s12906-0171990-y

Song WY, Ma YB, Bai X, Zhang XM, Gu Q, Zheng YT, Zhou J, Chen JJ (2007) Two new compounds and anti-HIV active constituents from Illicium verum. Planta Medica 73:372-375

Soo May L, Sanip Z, Ahmed Shokri A, Abdul Kadir A, Md Lazin MR (2018) The effects of Momordica charantia (bitter melon) supplementation in patients with primary knee osteoarthritis: A single-blinded, randomized controlled trial. Complementary Therapies in Clinical Practice 32:181-186

Srivastava KC, Mustafa T (1992) Ginger (Zingiber officinale) in rheumatism and musculo-skeletal disorders. Medical Hypotheses 39(4):342-348

Stajner D, Milic N, Canadanovic-Brunet J, Kapor A, Stajner M, Popovic BM (2006) Exploring Allium species as a source of potential medicinal agents. Phytotherapy Research 20:581-584

Stepp JR (2005) Advances in ethnobiological field methods. Field Methods 17:211-218

Su S, Wink M (2015) Natural lignans from Arctium lappaas antiaging agents in Caenorhabditis elegans. Phytochemistry 117:340-350

Sudjaroen Y (2013) Screening for antimicrobial and antimalarial activities of longan (Dimocarpus longan Lour) seeds. Scientific Research and Essays 8:917-920
Sukmandari NS, Dash GK, Jusof WHW, Hanafi M (2017) A Review on Nephelium lappaceum L. Research Journal of Pharmacy and Technology 10:2819-2827

Sun JY, You CY, Dong K, You HS, Xing JF (2016) Anti-inflammatory, analgesic and antioxidant activities of 3,4-oxo-isopropylidene-shikimic acid. Pharmaceutical Biology 54:2282-2287

Sundar Dhilip Kumar S, Houreld NN, Abrahamse $H$ (2018) Therapeutic potential and recent advances of curcumin in the treatment of aging-associated diseases. Molecules 23(4):E835, doi: 10.3390/molecules23040835

Sung YY, Yang WK, LeeAY, Kim DS, Nho KJ, Kim YS, Kim HK (2012a) Topical application of an ethanol extract prepared from Illicium verum suppresses atopic dermatitis in NC/Nga mice. Journal of Ethnopharmacology 144:151-159

Sung YY, Yang WK, Kim HK (2012b) Antiplatelet, anticoagulant and fibrinolytic effects of Litchi chinensis extract. Molecular Medicine Reports 5:721-724

Sung YY, Kim DS, Kim SH, Kim HK (2018) Aqueous and ethanolic extracts of welsh onion, Allium fistulosum, attenuate high-fat diet-induced obesity. BMC Complementary and Alternative Medicine 18(1):105, doi: 10.1186/s12906-018-2152-6

Szopa A, Ekiert R, Ekiert H (2017) Current knowledge of Schisandra chinensis (Turcz.) Baill. (Chinese magnolia vine) as a medicinal plant species: a review on the bioactive components, pharmacological properties, analytical and biotechnological studies. Phytochemistry Reviews 16:195-218

Tang X., Olatunji OJ, Zhou Y, Hou X (2017a) Allium tuberosum: Antidiabetic and hepatoprotective activities. Food Research International 102:681-689

Tang X, Olatunji OJ, Zhou Y, Hou X (2017b) In vitro and in vivo aphrodisiac properties of the seed extract from Allium tuberosum on corpus cavernosum smooth muscle relaxation and sexual behavior parameters in male Wistar rats. BMC Complementary and Alternative Medicine17(1):510, doi: 10.1186/s12906-017-2008-5

Thakur AK, Chatterjee SS, Kumar V (2014a) Beneficial effects of Brassica juncea on cognitive functions in rats. Pharmaceutical Biology 51:1304-1310 
Thakur AK, Chatterjee SS, Kumar V (2014b) Antidepressant-like effects of Brassica juncea L. leaves in diabetic rodents. Indian Journal of Experimental Biology 52:613-622

Thayyil AH, Surulivel MKM, Ahmed MF, Ahamed GSS, Sidheeq A, Rasheed A, Ibrahim M (2011) Hypolipidemic activity of Luffa aegiptiaca fruits in cholesterol fed hypercholeterolemic rabbits. International Journal of Pharmaceutical Applications 2:81-88.

The International Plant Names Index (2015) Database [https://www.ipni.org]. Accessed September 15th 2018

The Plant List (2013) The Plant List. Version 1.1. [http://www.theplantlist.org] Accessed October 15th 2018

Tian X, Sui S, Huang J, Bai JP, Ren TS, Zhao QC (2014) Neuroprotective effects of Arctium lappa L. roots against glutamate-induced oxidative stress by inhibiting phosphorylation of p38, JNK and ERK 1/2 MAPKs in PC12 cells. Environmental Toxicology and Pharmacology 38:189-198

Timité G, Mitaine-Offer AC, Miyamoto T, Tanaka C, Mirjolet JF, Duchamp O, Lacaille-Dubois MA (2013) Structure and cytotoxicity of steroidal glycosides from Allium schoenoprasum. Phytochemistry 88:61-66

Torabi M, Naeemzadeh $F$, Ebrahimi V, Taleschian-Tabrizi N, Pashazadeh F, Nazemie H (2017) The effect of Zingiber officinale (ginger) on hypertension. A systematic review of randomized controlled trials. BMJ Open 7(Suppl 1): doi: 10.1136/bmjopen-2016015415.133

Tseng HC, Wu WT, Huang HS, Wu MC (2014) Antimicrobial activities of various fractions of longan (Dimocarpus longan Lour.) seed extract. International Journal of Food Sciences and Nutrition 65:589-593

Tsuji R, Koizumi H, Fujiwara D (2011) Effects of a plum (Prunus mume Siebold \& Zucc.) ethanol extract on the immune system in vivo and in vitro. Bioscience, Biotechnology, and Biochemistry 75:2011-2013

Tsukayama I, Toda K, Takeda Y, Mega T, Tanaka M, Kawakami Y, Takahashi Y, Kimoto M, Yamamoto K, Miki Y, Murakami M, Suzuki-
Yamamoto $T$ (2018) Preventive effect of Dioscorea japonica on squamous cell carcinoma of mouse skin involving downregulation of prostaglandin E2 synthetic pathway. Journal of Clinical Biochemistry and Nutrition 62:139-147

Tyagi S, Chirag P, Dhruv M, Ishita M, Gupta A, Usman M, Nimbiwal B, Maheshwari R (2013) Medical benefits of Apium graveolens (celery herb). Journal of Drug Discovery and Therapeutics 1:36-38

Ueda H, Takeuchi A, Wako T (2013) Activation of immune responses in mice by an oral administration of bunching onion (Allium fistulosum) mucus. Bioscience, Biotechnology, and Biochemistry 77:1809-1813

Ueda K, Kawabata R, Irie T, Nakai Y, Tohya $Y$, Sakaguchi T (2013) Inactivation of pathogenic viruses by plant-derived tannins: strong effects of extracts from persimmon (Diospyros kaki) on a broad range of viruses. Plos One 8(1):e55343, doi: 10.1371/journal.pone.0055343

Une HD, Doshi GM (2016) Carissa congesta Wight and Benincasa hispida (Thunb.) Cogn. as budding immunomodulatory agents. Indian Journal of Experimental Biology 54:650-658

Vijaylakshmi P, Radha R (2015) An overview: Citrus maxima. Journal of Phytopharmacology 4:263-267

Volpato G, Godínez D, Beyra A (2009) Migration and ethnobotanical practices. The case of Tifey among Haitian immigrants in Cuba. Human Ecology 37:43-53

Wang BS, Huang GJ, Lu YH, Chang LW (2013) Anti-inflammatory effects of an aqueous extract of Welsh onion green leaves in mice. Food Chemistry 138:751-756

Wang GW, HuWT, Huang BK, Qin LP (2011) Illicium verum: a review on its botany, traditional use, chemistry and pharmacology. Journal of Ethnopharmacology 136:10-20

Wang GY, Yang C, Yang Z, Yang W, Jiang S, Zhang G, Guo Y, Wei M (2015) Effects of dietary star anise (Illicium verum Hook f.) supplementation during gestation and lactation on the performance of lactating multiparous sows and nursing piglets. Animal Science Journal 86:401-407 
Wang Z, Li P, Wang C, Jiang Q, Zhang L, Cao Y, Zhong W, Wang C (2016) Protective effects of Arctium lappa L. root extracts (AREs) on high fat diet induced quail atherosclerosis. BMC Complementary and Alternative Medicine16:6, doi: 10.1186/s12906-016-0987-2

Weil MJ, Zhang Y, Nair MG (2005) Tumor cell proliferation and cyclooxygenase inhibitory constituents in horseradish (Armoracia rusticana) and Wasabi (Wasabia japonica). Journal of Agricultural and Food Chemistry 53:1440-1444

Williams IO, Onyenweaku EO, Atangwho IJ (2016) Nutritional and antimicrobial evaluation of Saccharum officinarum consumed in Calabar, Nigeria. African Journal of Biotechnology 15:1789-1795

Wilson PB (2015) Ginger (Zingiber officinale) as an analgesic and ergogenic aid in sport: $A$ systemic review. Journal of Strength and Conditioning Research 29:2980-2995

Wojcik M, Krawczyk M, Wojcik P, Cypryk K, Wozniak LA (2018) Molecular mechanisms underlying curcumin-mediated therapeutic effects in Type 2 Diabetes and Cancer. Oxidative Medicine and Cellular Longevity 2018:9698258, doi: 10.1155/2018/9698258

Wu WH, Liu LY, Chung CJ, Jou HJ, Wang TA (2005) Estrogenic effect of Yam ingestion in healthy postmenopausal women. Journal of the American College of Nutrition 24:235-243

Wu ZY, Raven PH, Hong DY (1995-2013). Flora of China. Vol. 2-25 [http://www.efloras.org/flora_page.aspx?flora_id= 2] Accessed August 20th 2018

$\mathrm{Xu}$ JP (2018) Natural substances for cancer prevention. CRC Press, Boca Raton, USA

Yadav SP, Vats V, Ammini AC, Grover JK (2004) Brassica juncea (Rai) significantly prevented the development of insulin resistance in rats fed fructose-enriched diet. Journal of Ethnopharmacology 93:113-116

Yan XT, Lee SH, Li W, Jang HD, Kim YH (2015) Terpenes and sterols from the fruits of Prunus mume and their inhibitory effects on osteoclast differentiation by suppressing tartrate-resistant acid phosphatase activity. Archives of Pharmacal Research 8:186-192
Yang EJ, Kim SS, Moon JY, Oh TH, Baik JS, Lee $\mathrm{NH}$, Hyun CG (2010) Inhibitory effects of Fortunella japonica var. margarita and Citrus sunki essential oils on nitric oxide production and skin pathogens. Acta Microbiologica et Immunologica Hungarica 57:15-27

Yang WM, Shim KJ, Choi MJ, Park SY, Choi BJ, Chang MS, Park SK (2008) Novel effects of Nelumbo nucifera rhizome extract on memory and neurogenesis in the dentate gyrus of the rat hippocampus. Neuroscience Letters 443:104-107

Yang WS, Lee SR, Jeong YJ, Park DW, Cho YM, Joo HM, Kim I, Seu YB, Sohn EH, Kang SC (2016) Antiallergic activity of ethanol extracts of Arctium lappa L. undried roots and its active compound, oleamide, in regulating FceRI-mediated and MAPK signaling in RBL2H3 Cells. Journal of Agricultural and Food Chemistry 64:3564-3573

Yang X, Chen A, Ma Y, Gao Y, Gao Z (2003) Encyclopedic Reference of Traditional Chinese Medicine. Springer, Berlin, Germany

Yari S, Karamian R, Asadbegy M, Hoseini E, Moazzami Farida SH (2018) The protective effects of Arctium lappa $L$. extract on testicular injuries induced by ethanol in rats. Andrologia 13:e13086, doi: 10.1111/and.13086.

Yi LT, Li J, Su DX, Dong JF, Li CF (2012) Hypouricemic effect of the methanol extract from Prunus mume fruit in mice. Pharmaceutical Biology 50:1423-1427

Yilmaz N, Seven B, Timur H, Yorgancı A, İnal HA, Kalem MN, Kalem Z, Han Ö, Bilezikçi B (2018) Ginger (Zingiber officinale) female fertility: might improve. A rat model. Journal of the Chinese Medical Association 81(10):905-911

Yin J, Zhang H, Ye J (2008) Traditional Chinese Medicine in Treatment of Metabolic Syndrome. Endocrine, Metabolic and Immune Disorders-Drug Targets 8(2):99-111

Yokozawa T, Park CH, Noh JS, Roh SS (2014) Role of oligomeric proanthocyanidins derived from an extract of persimmon fruits in the oxidative stress-related aging process. Molecules 19:6707-6726 
Yoo JH, Yang KS (2012) Constituents of Pyrus pyrifolia with inhibitory activity on the NO production and the expression of iNOS and COX-2 in macrophages and microglia. Natural Product Sciences 18:183-189

Yu H, Qiu JF, Ma LJ, Hu YJ, Li P, Wan JB (2017) Phytochemical and phytopharmacological review of Perilla frutescens L. (Labiatae), a traditional edible-medicinal herb in China. Food and Chemical Toxicology 108(Pt B):375391

Yu SY, Gao R, Zhang L, Luo J, Jiang H, Wang S (2013) Curcumin ameliorates ethanol-induced memory deficits and enhanced brain nitric oxide synthase activity in mice. Progress in Neuro-Psychopharmacology and Biological Psychiatry 44:210-216

Yun JW, You JR, Kim YS, Kim SH, Cho EY, Yoon JH, Kwon E, Jang JJ, Park JS, Kim HC, Che JH, Kang BC (2018) In vitro and in vivo safety studies of cinnamon extract (Cinnamomum cassia) on general and genetic toxicology. Progress in Neuro-Psychopharmacology and Biological Psychiatry 95:115-123

Yuvakkumar R, Suresh J, Nathanael AJ, Sundrarajan M, Hong SI (2014) Novel green synthetic strategy to prepare ZnO nanocrystals using rambutan (Nephelium lappaceum L.) peel extract and its antibacterial applications. Materials Science and Engineering C: Materials for Biological Applications 41:17-27

Yuvakkumar R, Suresh J, Saravanakumar B, Joseph Nathanael A, Hong SI, Rajendran V (2015) Rambutan peels promoted biomimetic synthesis of bioinspired zinc oxide nanochains for biomedical applications. Spectrochimica Acta. A, Molecular and Biomolecular Spectroscopy 137:250-258

Zhang YJ, Zhou T, Wang F, Zhou Y, Li Y, Zhang JJ, Zheng J, Xu DP, Li HB (2016) The Effects of Syzygium samarangense, Passiflora edulis and Solanum muricatum on alcohol-induced liver injury. International Journal of Molecular Sciences 17(10):1616, doi: 10.3390/ijms 17101616
Zheng J, Zhou Y, Li Y, Xu DP, Li S, Li HB (2016) Spices for prevention and treatment of cancers. Nutrients 8(8):E495, doi: 10.3390/nu8080495

Zheng SQ, JiangF, Gao HY, Zheng JG (2010) Preliminary observations on the antifatigue effects of longan (Dimocarpus longan Lour.) seed polysaccharides. Phytotherapy Research 24:622-624

Zhou J, Xie G, Yan X (2011) Encyclopedia of Traditional Chinese Medicines. Molecular structures, pharmacological activities, natural sources and applications 5. Springer, Heidelberg, Germany

Zhou S, Ye Y, Afzan A, Allard PM, WolfrumC, Wolfender $J L$ (2016) Investigation of an edible TCM bitter melon (Momordica charantia): metabolite profiling, differentiation andcharacterization. Planta Medica 82(S01):S1-S381, doi: 10.1055/s-0036-1596193

Zhou YJ, Xiang JZ, Yuan H, Liu H, Tang Q, Hao HZ, Yin Z, Wang J, Ming ZY (2013) Neferine exerts its antithrombotic effect by inhibiting platelet aggregation and promoting dissociation of platelet aggregates. Thrombosis Research 132:202-210

Zhu J, Chen H, Song Z, Wang X, Sun Z (2018) Effects of ginger (Zingiber officinale Roscoe) on Type 2 Diabetes Mellitus and components of the Metabolic Syndrome: A systematic review and meta-analysis of randomized controlled trials. Evidence-Based Complementary and Alternative Medicine 2018:5692962, doi: 10.1155/2018/5692962

Zhu YP (1998) Chinese Materia Medica: Chemistry, Pharmacology and Applications. CRC Press, Boca Raton, USA

Received: 03 March 2019

Accepted: 12 July 2019

Published: 06 August 2019 